\title{
DERIVED EQUIVALENCE AND GROTHENDIECK RING OF VARIETIES: THE CASE OF K3 SURFACES OF DEGREE 12 AND ABELIAN VARIETIES
}

\author{
ATSUSHI ITO, MAKOTO MIURA, SHINNOSUKE OKAWA, AND KAZUSHI UEDA
}

\begin{abstract}
In this paper, we discuss the problem of whether the difference $[X]-[Y]$ of the classes of a Fourier-Mukai pair $(X, Y)$ of smooth projective varieties in the Grothendieck ring of varieties is annihilated by some power of the class $\mathbb{L}=\left[\mathbb{A}^{1}\right]$ of the affine line. We give an affirmative answer for Fourier-Mukai pairs of very general K3 surfaces of degree 12. On the other hand, we prove that in each dimension greater than one, there exists an abelian variety such that the difference with its dual is not annihilated by any power of $\mathbb{L}$, thereby giving a negative answer to the problem. We also discuss variations of the problem.
\end{abstract}

\section{Contents}

1. Introduction

Note added

Notation and conventions

Acknowledgements

2. K3 surfaces in $\mathrm{OG}(4,8)$

3. K3 surfaces in $\mathrm{OG}(5,10)$

4. Projective duality and derived equivalence

5. K3 surfaces in $\mathrm{G}(2,6)$

6. Abelian varieties

7. Variations of Problem 1.2

References

\section{INTRODUCTION}

Let $X$ and $Y$ be a pair of smooth projective varieties (or more generally smooth and proper Deligne-Mumford stacks) over a field $\mathbf{k}$. We say that $X$ is D-equivalent to $Y$ if the bounded derived category of coherent sheaves $D(X):=D^{b} \operatorname{coh} X$ is equivalent to $D(Y)$ as a k-linear triangulated category (we also say that $Y$ is a Fourier-Mukai partner of $X$ ).

It is shown in the pioneering paper Muk81] that an abelian variety is D-equivalent to its dual, meaning that non-birational varieties could be D-equivalent. The following natural question arises from this observation.

Question 1.1. Which piece of information of a variety (or more generally DeligneMumford stack) does the derived category have? In other words, if $X$ and $Y$ are Dequivalent, which invariants of $X$ and $Y$ do coincide?

It follows from the uniqueness of the Serre functor BK89 that $X$ and $Y$ have the same dimension and isomorphic (anti-)canonical rings. The Hochschild cohomology ring, whose graded pieces are the group of natural transformations from the identity functor 
to the shift functor, is also derived invariant. When the characteristic of the base field $\mathbf{k}$ is zero, combined with the Hochschild-Kostant-Rosenberg isomorphism [HKR62, Swa96, Yek02, Căl05, AC12, this gives the following partial coincidence of the Hodge numbers for any integer $i \in \mathbb{Z}$;

$$
\sum_{q-p=i} h^{p, q}(X)=\sum_{q-p=i} h^{p, q}(Y) .
$$

Another deep result is the coincidence of Chow motives with rational coefficients up to Tate twists Or105, Tab05, Kon09, Tab13.

On the other hand, Popa and Schnell [PS11] proved in characteristic zero that the identity components of the Picard schemes of $X$ and $Y$ are isogenous. As a corollary, it follows that the Hodge numbers of $X$ and $Y$ coincide for all $(p, q)$ under the assumption that $\operatorname{dim} X(=\operatorname{dim} Y) \leq 3$. Honigs [Hon18] also showed that, if $\mathbf{k}=\mathbb{F}_{q}$, the Hasse-Weil zeta functions coincide again in dimension 3 or less.

Recall that smooth projective varieties $X$ and $Y$ are $K$-equivalent if there is a diagram

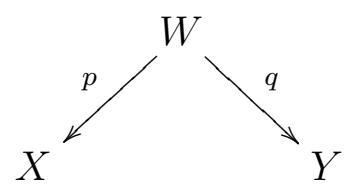

where $W$ is a normal variety and $p, q$ are birational projective morphisms satisfying $p^{*} K_{X} \simeq q^{*} K_{Y}$. It is conjectured and has been shown in some cases that K-equivalent varieties (and stacks) are D-equivalent. Conversely, it is also conjectured that birational and D-equivalent smooth projective varieties of non-negative Kodaira dimension are Kequivalent. For the details and the history of DK hypothesis, see the recent survey article [Kaw18] and references therein.

Combined with the arguments above, it is natural to ask if K-equivalent varieties have the same Hodge numbers when $\mathbf{k}$ has characteristic zero. In fact, this is known to be the case in its full generality. One way to prove this is to utilize the method of motivic integration, and here the Grothendieck ring of varieties comes into the picture.

The Grothendieck ring of varieties over a field $\mathbf{k}$, which will be denoted by $K_{0}(\mathcal{V}$ ar $/ \mathbf{k})$ in this paper, is the quotient of the free abelian group generated by the set of isomorphism classes of schemes of finite type over $\mathbf{k}$ modulo the relations

$$
[X]=[X \backslash Z]+[Z]
$$

for closed embeddings $Z \subset X$. Multiplication in $K_{0}(\mathcal{V}$ ar $/ \mathbf{k})$ is defined by the Cartesian product, which is easily seen to be associative, commutative, and unital with $1=$ [Spec $\mathbf{k}]$.

When $\mathbf{k}$ is of characteristic zero, it is shown by means of the motivic integration of Kontsevich [Bat98, DL99] that classes of smooth projective K-equivalent varieties coincide in the completed Grothendieck ring of varieties. This is a completion of the localized Grothendieck ring $K_{0}(\mathcal{V}$ ar $/ \mathbf{k})\left[\mathbb{L}^{-1}\right]$, where $\mathbb{L}=\left[\mathbb{A}^{1}\right]$ is the class of the affine line (see Definition 6.1). From this result, by taking (an extension of) the Hodge-Deligne polynomial, one immediately obtains the coincidence of the Hodge numbers for K-equivalent varieties.

In view of this result, it is natural to ask if D-equivalent varieties have the same class in the completed (or localized) Grothendieck ring of varieties.

Problem 1.2 ([IMOU, Problem 1.3]). Let $(X, Y)$ be a Fourier-Mukai pair. Does the equality

$$
([X]-[Y]) \cdot \mathbb{L}^{k}=0 \in K_{0}(\mathcal{V a r} / \mathbf{k})
$$


hold for a non-negative integer $k$ ?

For example, one can take $k=0$ if $X$ and $Y$ are related by an elementary flop appearing in $[\mathrm{BO}]$.

Recently a number of positive results have been obtained by several groups of people.

(1) Borisov [Bor18] show that the Pfaffian-Grassmannian pairs $(X, Y)$ of Calabi-Yau 3-folds [Rød00] satisfy

$$
([X]-[Y])\left(\mathbb{L}^{2}-1\right)(\mathbb{L}-1) \mathbb{L}^{7}=0
$$

in $K_{0}(\mathcal{V}$ ar $/ \mathbf{k})$, giving a first counter-example to the cancellation problem, which asks the injectivity of the homomorphism $K_{0}(\mathcal{V}$ ar $/ \mathbf{k}) \rightarrow K_{0}(\mathcal{V}$ ar $/ \mathbf{k})\left[\mathbb{L}^{-1}\right]$. (1.5) is subsequently refined by Martin [Mar16] to

$$
([X]-[Y]) \cdot \mathbb{L}^{6}=0 .
$$

(2) It is shown in [MOU19 that for pairs $\left(X^{\prime}, Y^{\prime}\right)$ of smooth Calabi-Yau 3-folds obtained as certain degenerations of $X$ and $Y$, one has

$$
\left(\left[X^{\prime}\right]-\left[Y^{\prime}\right]\right) \cdot \mathbb{L}=0 .
$$

Both the Pfaffian-Grassmannian pairs $(X, Y)$ of Calabi-Yau 3-folds and their degenerations $\left(X^{\prime}, Y^{\prime}\right)$ are Fourier-Mukai partners by [BC09, Kuz and [Kuz18] (see also [Ued19]).

(3) In [KS], Kuznetsov and Shinder studied the Fourier-Mukai partners of K3 surfaces of degree 8 and 2. A K3 surface $X$ is said to be of degree $d$ if there is an ample line bundle $L$ on $X$ satisfying $L \cdot L=d$. They prove that there are such pairs $\left(X^{\prime \prime}, Y^{\prime \prime}\right)$ satisfying

$$
\left(\left[X^{\prime \prime}\right]-\left[Y^{\prime \prime}\right]\right) \cdot \mathbb{L}=0 .
$$

(4) In [BCP17, OR], it is shown that a generic pair of the so-called GPK ${ }^{3}$ Calabi-Yau 3 -folds $\left(X^{\prime \prime \prime}, Y^{\prime \prime \prime}\right)$ are D-equivalent, non-birational, and satisfy the equality

$$
\left(\left[X^{\prime \prime \prime}\right]-\left[Y^{\prime \prime \prime}\right]\right) \cdot \mathbb{L}^{4}=0 .
$$

See also [KR17] for further study in this direction.

(5) Examples of pairs of K3 surfaces of degree 2 are given in KKM17, Proposition 4.1].

(6) A series of examples consisting of hyperkähler manifolds of $K 3[n]$-type is given in Oka18] (see also [MMY19] for finer results on birational (in)equivalence of those manifolds).

(7) Examples of Calabi-Yau 5-folds are given in [Man19].

(8) Further examples are given in [SZ19].

Problem 1.2 was stated as [KS, Conjecture 1.6] around the same time as [IMOU], the first preprint version of this paper.

One of the purposes of this paper is, as an application of the geometry of equivariant vector bundles on homogeneous spaces of type $D$, to prove that a general Fourier-Mukai pair of K3 surfaces of degree 12 gives another example of an affirmative answer to Problem 1.2 .

Theorem 1.3. Let $X$ be a general K3 surface of degree 12 over $\mathbb{C}$. Then there exists a non-isomorphic Fourier-Mukai partner $Y$ of $X$ satisfying

$$
([X]-[Y]) \cdot \mathbb{L}^{3}=0 .
$$


Remark 1.4. After the completion of the first draft of this paper, Brendan Hassett and Kuan-Wen Lai proved in [HL, Theorem 4.1] the stronger equality

$$
([X]-[Y]) \cdot \mathbb{L}=0 \in K_{0}(\mathcal{V a r} / \mathbb{C}),
$$

by a completely different method based on Cremona transformations of $\mathbb{P}^{4}$.

It follows from [Ogu02, Proposition 1.10] (see also [HLOY04, Corollary 2.7.4]) that the number of isomorphism classes of Fourier-Mukai partners of a K3 surface over $\mathbb{C}$ with Picard number 1 and degree 12 is 2. Hence Theorem 1.3 and [HL, Theorem 4.1] gives an affirmative answer to Problem 1.2 for very general K3 surfaces of degree 12 over $\mathbb{C}$.

Our second purpose, however, is to explain that there are examples which negatively answer to Problem 1.2.

Theorem 1.5. If $A$ is a complex abelian variety which is not isomorphic to its dual $\widehat{A}$, then $[A] \neq[\widehat{A}]$ in the completed Grothendieck ring of varieties $\widehat{K}_{0}(\mathcal{V}$ ar $/ \mathbb{C})$. In particular, for any integer $g \geq 2$ there exists a pair of non-isomorphic complex abelian $g$-folds which are D-equivalent but have distinct classes in $\widehat{K}_{0}(\mathcal{V}$ ar $/ \mathbb{C})$.

The same example is also discovered independently in [Efi18, Theorem 3.1]. Our proof uses Proposition 6.3 (= Eke09, Proposition 3.6]), which is based on the solution of Tate isogeny theorem by Faltings. In [Efi18], this part is replaced with more elementary categorical arguments.

In Corollary 6.7, we explain that the pair of Hilbert schemes of points $\left(A^{[n]}, B^{[n]}\right)$, where $(A, B)$ is a pair of abelian surfaces as in Theorem 1.5, is another example of a negative answer to Problem 1.2

Because of these negative examples, Kuznetsov and Shinder modified their conjecture in the published version [KS18, Conjecture 1.6] by adding the extra assumption that $X$ and $Y$ should be simply connected. However, it is desirable to modify Problem 1.2 in such a way that it still has meaningful implications such as the coincidence of the Hodge numbers over a field of characteristic zero and that of the number of rational points over a finite field, without adding extra assumptions on the pair $(X, Y)$. In Section [7, we raise problems in this direction.

Another interesting problem is to extend the whole picture in such a way that not only equivalences but also admissible embeddings of triangulated categories are taken into account. Let $\Gamma_{\mathbf{k}}$ be the ring defined in [BLL04, Definition 8.1] as the quotient of the free abelian group generated by quasi-equivalence classes of enhanced (=pretriangulated $\mathrm{dg}$ ) bounded derived categories $\mathscr{D}(X)$ of smooth complex projective varieties $X$ by the relations

$$
[\mathscr{D}(X)]=\left[\mathscr{D}\left(Y_{1}\right)\right]+\cdots+\left[\mathscr{D}\left(Y_{n}\right)\right]
$$

for semiorthogonal decompositions

$$
\mathscr{D}(X)=\left\langle\mathscr{B}_{1}, \ldots, \mathscr{B}_{n}\right\rangle
$$

with $\mathscr{D}\left(Y_{i}\right) \simeq \mathscr{B}_{i}$ for $i=1, \ldots, n$. Multiplication in $\Gamma_{\mathbf{k}}$ is defined by the tensor product of dg categories. It is shown in [BLL04, Section 8] that there exists a motivic measure

$$
K_{0}(\mathcal{V a r} / \mathbf{k}) \rightarrow \Gamma_{\mathbf{k}}
$$

sending the class $[X]$ of a smooth projective variety to $[\mathscr{D}(X)]$, which descends to a ring homomorphism

$$
K_{0}(\mathcal{V} \text { ar } / \mathbf{k}) /(\mathbb{L}-1) \rightarrow \Gamma_{\mathbf{k}}
$$


Problem 1.2 is closely related to the problem, given implicitly at the end of [BLL04, Section 8], of asking how close to being injective the map (1.15) is.

This paper is organized as follows: In Section 2, we give pairs $(X, Y)$ of Calabi-Yau manifolds defined as zeros of sections of equivariant vector bundles on homogeneous spaces of $\operatorname{Spin}(2 m)$, and prove the equality (2.13) in the Grothendieck ring of varieties. By specializing to $m=4$, we obtain pairs of K3 surfaces of degree 12. In Section 3, we show that the K3 surfaces can be also described as linear sections of a homogeneous space OG $(5,10)$ of Spin(10). In Section 4, we use results of Mukai to complete the proof of Theorem 1.3. In Section 5, we give a formula in the Grothendieck ring of varieties which describes the class of Pfaffian cubic 4-folds by the class of the associated K3 surfaces and $\mathbb{L}$. This is compatible with the homomorphism (1.15), in that the terms in the formula are sent to the SOD summands. In Section 6, we discuss negative answers to Problem 1.2. In Section 7, we discuss variations of Problem 1.2.

Note added. After the completion of Section 7 and before it was made public, the preprint [Bal19] appeared on the arXiv, which has some overlap with Section 7 . In particular, our Proposition 7.1 is essentially the same as [Bal19, Theorem 1.6]. Compared to us, the author of [Bal19] seems more concentrated on the relationship between $D$ equivalence and Chow motives (see [Bal19, Propositions 1.7, 1.8]).

Notation and conventions. Throughout the paper, we use the dual of the Grothendieck's convention for projectivization. Namely, for a locally free sheaf $\mathcal{F}$ on a scheme $X$, we write $\mathbb{P}_{X}(\mathcal{F})$ to mean $\operatorname{Proj}_{X} \operatorname{Sym} \mathcal{F}^{\vee}$. In particular, for $X=\operatorname{Spec} \mathbf{k}$ and $V=\mathcal{F}$, the k-rational points of the scheme $\mathbb{P}(V)=G(1, V)$ represent lines of $V$.

Acknowledgements. We thank Kenji Hashimoto and Daisuke Inoue for collaboration at an early stage of this work; this note is originally conceived as a joint project with them. We also thank Genki Ouchi for Remark 5.1 and the reference [BLL04], and Kota Yoshioka for the reference [HLOY03]. We also thank Yujiro Kawamata, Keiji Oguiso, Evgeny Shinder, Hokuto Uehara, and Takehiko Yasuda for useful discussions. We also thank the anonymous referees for reading the manuscript carefully and suggesting a number of improvements. A. I. was supported by Grants-in-Aid for Scientific Research (14J01881,17K14162). M. M. was supported by Korea Institute for Advanced Study. S. O. was partially supported by Grants-in-Aid for Scientific Research (16H05994, 16K13746, 16H02141, 16K13743, 16K13755, 16H06337, 18H01120) and the Inamori Foundation. K. U. was partially supported by Grants-in-Aid for Scientific Research (24740043, 15KT0105, 16K13743, 16H03930).

\section{K3 SURFACES IN OG(4,8)}

Let $V_{0}$ be a vector space of dimension $m$, so that $V_{\text {nat }}:=V_{0} \oplus V_{0}^{\vee}$ is a vector space of dimension $2 m$ equipped with the natural non-degenerate bilinear form

$$
\left\langle v+\check{v}, v^{\prime}+\check{v}^{\prime}\right\rangle=\check{v}\left(v^{\prime}\right)+\check{v}^{\prime}(v) .
$$

The spin group $G:=G_{m}:=\operatorname{Spin}\left(V_{\text {nat }}\right)$ is the simply-connected simple algebraic group, which is obtained as the double cover of $\mathrm{SO}\left(V_{\text {nat }}\right)$. The spinor representation is a $2^{m}$ dimensional representation of $G$ on $\Lambda V_{0}$, which decomposes as the direct sum of half spinor representations $V_{1}:=\bigwedge^{\text {even }} V_{0}$ and $V_{2}:=\bigwedge^{\text {odd }} V_{0}$. These half spinor representations are related to each other by an outer automorphism of $\operatorname{Spin}(2 m)$. They are self-dual if $m$ is even, and dual to each other if $m$ is odd. Let $\omega_{1}$ and $\omega_{2}$ be the fundamental weights corresponding to the half spinor representations $V_{1}$ and $V_{2}$ respectively. Given a dominant 
integral weight $\lambda$ of $G$, the irreducible representation of $G$ with highest weight $\lambda$ will be denoted by $V_{\lambda}$. We also write $(i, j):=i \omega_{1}+j \omega_{2}$, so that $V_{1}=V_{(1,0)}$ and $V_{2}=V_{(0,1)}$.

Let $F_{i}$ be the homogeneous space of $G$ associated with the Dynkin diagram

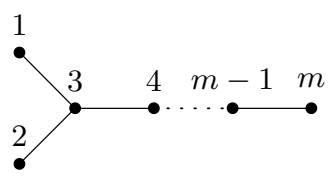

of type $D_{m}$ with the $i$-th node crossed out (see [BE89, Section 2.3] for the notion and rudiments of crossed Dynkin diagrams). The nodes of the Dynkin diagram correspond to the simple roots, and the fundamental weights $\omega_{1}, \ldots, \omega_{m}$. In this paper we use the numbering of nodes as in (2.2). For $i=3, \ldots, m$, the numbering fits well with the description of $F_{i}$ as orthogonal Grassmannians:

$$
\begin{aligned}
F_{i} & =\mathrm{OG}\left(m-i+1, V_{\text {nat }}\right) \\
& :=\left\{V \subset V_{\text {nat }}|\langle-,-\rangle|_{V}=0 \text { and } \operatorname{dim} V=m-i+1\right\} .
\end{aligned}
$$

We also let $\mathrm{OG}\left(m, V_{\text {nat }}\right)$ and $\mathrm{OG}(m, 2 m)$ denote one of the connected components of the space (2.3) for $i=1$, which coincides with the spinor variety $F_{1} \simeq F_{2}$. Let further $F_{1,2}$ be the homogeneous space of $G$ associated with the Dynkin diagram (2.2) with the nodes 1 , 2 crossed out. It can naturally be identified with $\mathrm{OG}\left(m-1, V_{\text {nat }}\right) \simeq \mathbb{P}_{F_{i}}\left(\mathcal{S}_{i}^{\vee}\right)$ for $i=1,2$, where $\mathcal{S}_{i}$ is the tautological subbundle of $V_{\text {nat }} \otimes_{\mathbf{k}} \mathcal{O}_{F_{i}}$ on $F_{i}$. They fit into the following diagram:

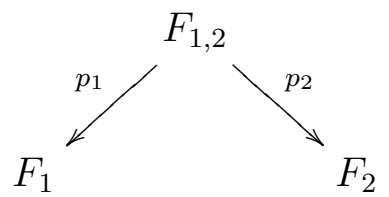

The Picard group of $F_{1,2}$ is given by

$$
\operatorname{Pic}\left(F_{1,2}\right)=p_{1}^{*} \operatorname{Pic}\left(F_{1}\right) \oplus p_{2}^{*} \operatorname{Pic}\left(F_{2}\right) \simeq \mathbb{Z}^{2} .
$$

For $i=1,2$, we let $\mathcal{O}_{F_{i}}(1)$ denote the ample generator of Pic $\left(F_{i}\right)$. Then the line bundle $\mathcal{O}_{F_{1,2}}(1,1):=\mathcal{O}_{F_{1}}(1) \otimes \mathcal{O}_{F_{2}}(1)$ is very ample, and one has

$$
F_{1,2} \simeq \mathbb{P}_{F_{i}}\left(\left(p_{i *} \mathcal{O}_{F_{1,2}}(1,1)\right)^{\vee}\right)
$$

for both $i=1,2$. Recall that homogeneous vector bundles on $F_{i}$ correspond to representations of the parabolic subgroup $P_{i} \subset G$ corresponding to $F_{i}$, which in turn correspond to representations of the Levi subgroup of $P_{i}$ if they are irreducible representations. In our case the Levi subgroup is isomorphic to $S(\mathrm{GL}(m) \times \mathrm{GL}(1))$, where $S($ ) denotes the subgroup consisting of elements of determinant 1 . In this sense $p_{i *} \mathcal{O}_{F_{1,2}}(1,1)$ is the locally free sheaf of rank $m$ on $F_{i}$ associated with the representation of $S(\mathrm{GL}(m) \times \mathrm{GL}(1))$ with highest weight $(1,1)$. If $i$ and $j$ are non-negative integers, then one has

$$
H^{0}\left(\mathcal{O}_{F_{1,2}}(i, j)\right) \simeq V_{(i, j)}^{\vee}
$$

by the Borel-Weil theorem.

Let

$$
s \in H^{0}\left(F_{1,2}, \mathcal{O}_{F_{1,2}}(1,1)\right) \simeq H^{0}\left(F_{1}, p_{1 *} \mathcal{O}_{F_{1,2}}(1,1)\right) \simeq H^{0}\left(F_{2}, p_{2 *} \mathcal{O}_{F_{1,2}}(1,1)\right)
$$


be a general section and let

$$
\begin{aligned}
D & :=Z(s) \subset F_{1,2}, \\
X & :=Z\left(p_{1 *} s\right) \subset F_{1}, \\
Y & :=Z\left(p_{2 *} s\right) \subset F_{2},
\end{aligned}
$$

be its zero loci, which are smooth complete intersections by Bertini Muk92, Theorem 1.10]. One can easily compute the rank and the degree of $p_{i_{*}} \mathcal{O}_{F_{1,2}}(1,1)$ to show that $X$ and $Y$ are Calabi-Yau of dimension $m(m-3) / 2$. Set $\pi_{i}=\left.p_{i}\right|_{D}: D \rightarrow F_{i}$ for $i=1,2$.

Lemma 2.1. The morphism $\pi_{1}$ is a $\mathbb{P}^{m-2}$-bundle over $F_{1} \backslash X$ and a $\mathbb{P}^{m-1}$-bundle over $X$, which are locally trivial in the Zariski topology. The same holds for $\pi_{2}$.

Proof. Since the arguments for $\pi_{1}$ and $\pi_{2}$ are the same, we only give it for $\pi_{1}$. Fix a point $x \in F_{1}$. Since $p_{1}$ is a projective bundle and $\mathcal{O}_{F_{1,2}}(1,1)$ is $p_{1}$-ample, by cohomology-andbase-change, we obtain the standard isomorphism

$$
\left.p_{1 *} \mathcal{O}_{F_{1,2}}(1,1)\right|_{x} \stackrel{\sim}{\rightarrow} H^{0}\left(p_{1}^{-1}(x),\left.\mathcal{O}_{F_{1,2}}(1,1)\right|_{p_{1}^{-1}(x)}\right)
$$

sending $\left.\left(p_{1 *} s\right)(x) \in p_{1 *} \mathcal{O}_{F_{1,2}}(1,1)\right|_{x}$ to $\left.s\right|_{p_{1}^{-1}(x)}$. Hence

$$
D \cap p_{1}^{-1}(x)=\pi_{1}^{-1}(x)
$$

is isomorphic to $p_{1}^{-1}(x) \simeq \mathbb{P}^{m-1}$ if $x \in X$, and to a hyperplane therein otherwise. It follows that the short exact sequence

$$
0 \rightarrow \mathcal{O}_{F_{1}} \stackrel{p_{1 *} s}{\rightarrow} p_{1 *} \mathcal{O}_{F_{1,2}}(1,1) \rightarrow p_{1 *} \mathcal{O}_{F_{1,2}}(1,1) / \mathcal{O}_{F_{1}}=: \mathcal{Q} \rightarrow 0
$$

splits Zariski locally on $F_{1} \backslash X$, so that $\pi_{1}$ is the $\mathbb{P}^{m-2}$ bundle over $F_{1} \backslash X$ associated to the locally free sheaf $\mathcal{Q}$. The latter claim follows from $\left.s\right|_{p_{1}^{-1}(X)}=0$.

Corollary 2.2. One has

$$
([X]-[Y]) \mathbb{L}^{m-1}=0
$$

in the Grothendieck ring of varieties.

Proof. One has

$$
[D]=\left(\left[F_{1}\right]-[X]\right)\left[\mathbb{P}^{m-2}\right]+[X]\left[\mathbb{P}^{m-1}\right]=\left(\left[F_{2}\right]-[Y]\right)\left[\mathbb{P}^{m-2}\right]+[Y]\left[\mathbb{P}^{m-1}\right]
$$

by Lemma 2.1. Since $F_{1}$ and $F_{2}$ are related by an outer automorphism of $\operatorname{Spin}(2 m)$ and hence isomorphic as algebraic varieties, one has

$$
0=([X]-[Y])\left(\left[\mathbb{P}^{m-1}\right]-\left[\mathbb{P}^{m-2}\right]\right)=([X]-[Y]) \mathbb{L}^{m-1},
$$

and Corollary 2.2 is proved.

Remark 2.3. Let $\mathrm{G}\left(m, V_{\text {nat }}\right)$ be the Grassmannian of $m$-spaces in $V_{\text {nat }}=V_{0} \oplus V_{0}^{\vee}$, and $\mathcal{S}$ be the universal subbundle on it. The zero of the section $s^{\prime}$ of $\mathrm{Sym}^{2} \mathcal{S}^{\vee}$ associated with the natural pairing $\langle-,-\rangle$ on $V_{\text {nat }}$ is the homogeneous space of the form

$$
\mathrm{O}\left(V_{\text {nat }}\right) / \mathrm{GL}\left(V_{0}\right) \text {. }
$$

It has two connected components, which can be identified with $F_{1}$ and $F_{2}$ in such a way that $V_{\text {nat }}=V_{\omega_{m}}$ as a $G$-module, and $\left.\mathcal{S}^{\vee}\right|_{F_{i}}$ is the equivariant vector bundle associated with the irreducible representation of $P_{i}$ with the highest weight $\omega_{m}$ for both $i=1$ and 2 . Note that the lowest weight of this irreducible representation of $P_{1}$ (resp. $P_{2}$ ) is $\omega_{1}-\omega_{2}$ (resp. $\left.-\omega_{1}+\omega_{2}\right)$, so that $\left.\mathcal{S}\right|_{F_{1}}$ (resp. $\left.\mathcal{S}\right|_{F_{2}}$ ) is the equivariant vector bundle associated with the irreducible representation of $P_{1}$ (resp. $P_{2}$ ) with the highest weight $-\omega_{1}+\omega_{2}$ 
(resp. $\left.\omega_{1}-\omega_{2}\right)$. Since the ample generator of Pic G( $\left.m, V_{\text {nat }}\right)$ restricts to twice the ample generator of $\mathrm{Pic} F_{1}$, one has

$$
\begin{aligned}
\left.\mathcal{S}(1)\right|_{F_{1}} & \simeq p_{1 *} \mathcal{O}_{F_{1,2}}(-1,1) \otimes p_{1 *} \mathcal{O}_{F_{1,2}}(2,0) \\
& \simeq p_{1_{*}} \mathcal{O}_{F_{1,2}}(1,1) .
\end{aligned}
$$

The same reasoning for $F_{2}$ gives

$$
\left.\mathcal{S}(1)\right|_{F_{2}}=p_{2 *} \mathcal{O}_{F_{1,2}}(1,1) .
$$

Since $H^{0}(\mathcal{S}(1)) \rightarrow H^{0}\left(F_{1},\left.\mathcal{S}(1)\right|_{F_{1}}\right)$ is a projection to an irreducible component of restricted representation by the Borel-Weil theorem, the section $s$ in (2.8) is extended as an element of $H^{0}(\mathcal{S}(1))$. Therefore, it follows that the zero of the section

$$
s+s^{\prime} \in H^{0}\left(\mathcal{S}(1) \oplus \operatorname{Sym}^{2} \mathcal{S}^{\vee}\right)
$$

is isomorphic to the disjoint union of $X$ and $Y$.

Remark 2.4. When $m=4$, the homogeneous spaces $F_{1}$ and $F_{2}$ are related to $F_{4}$ by an outer automorphism of $\operatorname{Spin}(8)$ called the triality automorphism. The homogeneous space $F_{4}=\mathrm{OG}\left(1, V_{\text {nat }}\right)$ is a quadric hypersurface in $\mathrm{G}\left(1, V_{\text {nat }}\right) \simeq \mathbb{P}^{7}$, and $X$ and $Y$ are K3 surfaces of degree 12.

\section{K3 SURfaCES IN OG(5,10)}

We use the same notation as in Section 2, We write $\mathcal{O}_{F_{1}}(i):=p_{1 *} \mathcal{O}_{F_{1,2}}(i, 0)$ and $\mathcal{E}:=p_{1 *} \mathcal{O}_{F_{1,2}}(0,1)$, so that $H^{0}\left(\mathcal{O}_{F_{1}}(1)\right) \simeq V_{1}^{\vee}$ and $H^{0}(\mathcal{E}) \simeq V_{2}^{\vee}$. Let

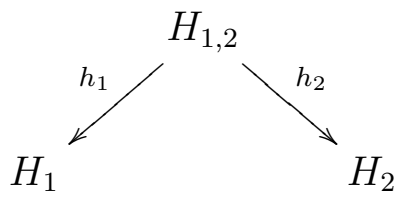

be the diagram obtained by replacing $m$ with $m+1$ in the diagram (2.4). We use the same numbering $1,2, \ldots, m+1$ for the nodes of the Dynkin diagram $D_{m+1}$ as that of $D_{m}$ in Section 2. In some places we will write $G_{m+1}$ to mean the spin group of type $D_{m+1}$, to avoid confusion. Lemma 3.1 below is an adaptation of [IIM19, Lemma 4.2].

Lemma 3.1. Let $F_{1} \subset H_{1}$ be the equivariant embedding corresponding to the unique inclusion of the Dynkin diagram of type $D_{m}$ with the node 1 crossed out into that of type $D_{m+1}$ with the same node crossed out.

(1) The following morphism is surjective.

$$
\left.h_{2}\right|_{h_{1}^{-1}\left(F_{1}\right)}: h_{1}^{-1}\left(F_{1}\right) \rightarrow H_{2}
$$

(2) There exists an isomorphism of projective bundles over $F_{1}$ as follows.

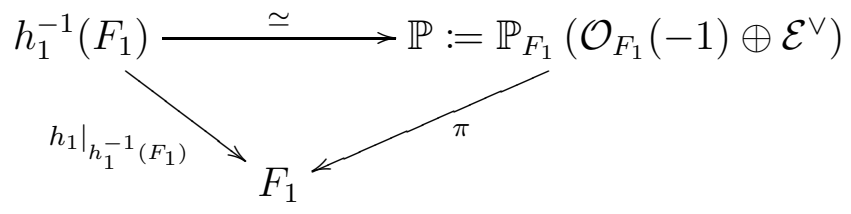

Proof. The argument is almost the same as the proof of [IM19, Lemma 4.2], except for some computations relevant to Tits transformations and equivariant vector budles. Hence we will be brief. 
Consider the following diagram, which corresponds to [IIM19, (75)].

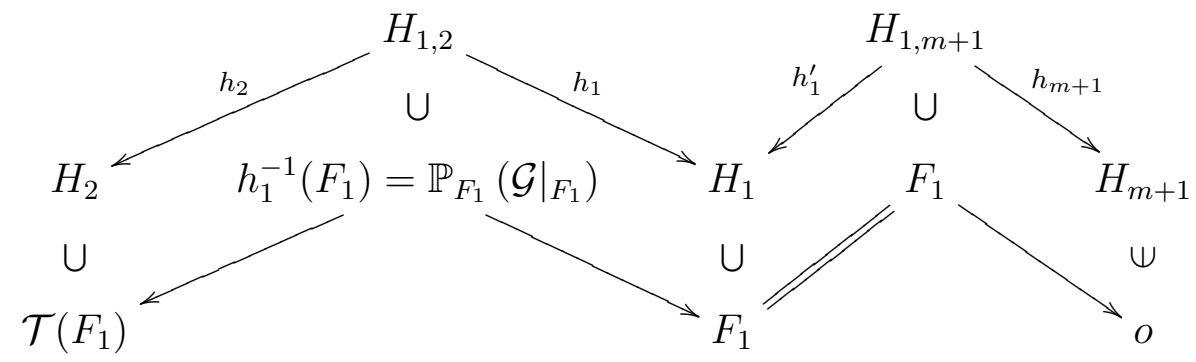

This diagram is obtained in the following way. Fix a Borel subgroup $B$ of $G_{m+1}=$ $\operatorname{Spin}(2 m+2)$, and let $B \subset P_{i} \subset G_{m+1}$ be the maximal parabolic subgroups corresponding to the fundamental weight $\omega_{i}$ for $i=1, \ldots, m+1$. We will write $H_{i}:=G_{m+1} / P_{i}$ and $H_{i, j}:=G_{m+1} /\left(P_{i} \cap P_{j}\right)$, consistently with the notation of (3.1). Let $W$ and $W_{P_{i}}$ denote the Weyl groups of $G$ and $P_{i}$, respectively. Let further $W^{P_{i}} \subset W$ be the set of minimal length representatives of the coset $W_{P_{i}} \backslash W$ for $i=1, \ldots, m+1$.

To show the assertion (1), we apply [CR13, Lemma 2.4] twice to the diagram (3.4); first to the Tits transform $F_{1}=\mathcal{T}(o):=h_{1}^{\prime}\left(h_{m+1}^{-1}(o)\right)$ of the point $o:=P_{m+1} / P_{m+1}$, which is induced by the right hut in the diagram, and then to the Tits transform $\mathcal{T}\left(F_{1}\right):=$ $h_{2}\left(h_{1}^{-1}\left(F_{1}\right)\right)$ induced by the left hut. Each of the two transformations is a correspondence of Schubert cycles, hence is described as the correspondence of (representatives of) the appropriate cosets of the Weyl groups. Concretely, the correspondences for the first and the second transformations are given by the composition of the following maps for $(i, j)=(m+1,1)$ and $(1,2)$ respectively, where $w_{i}$ denotes the longest elements of $W_{P_{i}}$ for all $i$.

$$
t_{i, j}: W^{P_{i}} \rightarrow w_{i} W^{P_{i}} \subset W \rightarrow W_{P_{j}} \backslash W \simeq W^{P_{j}}
$$

Together with Lemma 3.2 below, we have

$$
t_{m+1,1}(\mathrm{id})=\widetilde{w}_{m+1} \text { and } t_{1,2}\left(\widetilde{w}_{m+1}\right)=w^{P_{2}},
$$

where $\widetilde{w}_{m+1} \in W^{P_{1}}$ be the minimal length representative of $W_{P_{1}} w_{m+1} \in W_{P_{1}} \backslash W$, and $w^{P_{i}}$ denotes the longest element of $W^{P_{i}}$ for all $i$. Therefore $F_{1}$ and $\mathcal{T}\left(F_{1}\right)$ turn out to be the Schubert varieties

$$
\overline{B \widetilde{w}_{m+1}^{-1} P_{1} / P_{1}} \subset H_{1} \text { and } X\left(w^{P_{2}}\right):=\overline{B\left(w^{P_{2}}\right)^{-1} P_{2} / P_{2}} \subset H_{2},
$$

respectively. Since $\operatorname{dim} X\left(w^{P_{2}}\right)=l\left(w^{P_{2}}\right)$ is the maximum, we obtain $\mathcal{T}\left(F_{1}\right)=X\left(w^{P_{2}}\right)=$ $\mathrm{H}_{2}$.

For (2), we consider two equivariant vector bundles $\mathcal{G}^{\vee}$ over $H_{1}$ and $\mathcal{G}^{\prime \vee}$ over $H_{1, m+1}$, both of which correspond to the irreducible representations of the highest weight $\omega_{2}$, i.e., $H^{0}\left(H_{1}, \mathcal{G}^{\vee}\right) \simeq H^{0}\left(H_{1, m+1}, \mathcal{G}^{\vee \vee}\right) \simeq V_{2}^{\vee}$ in the sense of $G_{m+1}$-modules. Since $h_{1}^{-1}\left(F_{1}\right)=$ $\mathbb{P}_{F_{1}}\left(\left.\mathcal{G}\right|_{F_{1}}\right)$, it is enough to show $\left.\mathcal{G}\right|_{F_{1}}=\mathcal{O}_{F_{1}}(-1) \oplus \mathcal{E}^{\vee}$. As we can see in the same way as [IIM19, (77)], we have a short exact sequence

$$
\left.\left.\left.0 \rightarrow \mathcal{G}^{\prime}\right|_{F_{1}} \rightarrow\left(h_{1}^{\prime *} \mathcal{G}\right)\right|_{F_{1}} \simeq \mathcal{G}\right|_{F_{1}} \rightarrow \mathcal{O}_{F_{1}}(-1) \rightarrow 0
$$

Note that there is an equivalence of categories

$$
\operatorname{VB}_{G}(G / P) \simeq \bmod (P) ;\left.\quad \mathcal{N} \mapsto \mathcal{N}\right|_{P / P}
$$

between the category of $G$-equivariant vector bundles on $G / P$ and the category of $P$ modules, for any parabolic subgroup $P \subset G$. Under this equivalence, $\left.\mathcal{G}^{\prime \mathrm{V}}\right|_{F_{1}}$ corresponds to the $\left(P_{1} \cap P_{m+1}\right)$-module of highest weight $\omega_{2}$ regarded as a $\left(P_{1} \cap \operatorname{Spin}(2 m)\right)$-module. Since this is the $\left(P_{1} \cap \operatorname{Spin}(2 m)\right)$-module of highest weight $\omega_{2}$, we have $\left.\mathcal{G}^{\prime}\right|_{F_{1}} \simeq \mathcal{E}^{\vee}$. 
Moreover, $\mathcal{E}^{\vee}(1)$ corresponds to the irreducible $\left(P_{1} \cap \operatorname{Spin}(2 m)\right)$-module whose highest weight is the dominant weight $\omega_{m+1}$. Hence, by the Bott-Borel-Weil theorem, we obtain

$$
\operatorname{Ext}^{1}\left(\mathcal{O}_{F_{1}}(-1), \mathcal{E}^{\vee}\right)=H^{1}\left(\mathcal{E}^{\vee}(1)\right)=0 \text {. }
$$

Thus we conclude the proof.

Lemma 3.2. Let us use the same notation in the proof of Lemma 3.1. It holds

$$
W_{P_{2}} w_{1} \widetilde{w}_{m+1}=W_{P_{2}} w^{P_{2}} .
$$

Proof. One of a reduced decomposition of the longest element of the Weyl group of each type is given by Lit98. In particular, we have

$$
\begin{aligned}
w_{1} & =\left(s_{2}\right)\left(s_{3} s_{2}\right)\left(s_{4} s_{3} s_{2}\right)(\ldots)\left(s_{m+1} \ldots s_{4} s_{3} s_{2}\right), \\
w_{m+1} & =\left(s_{1} s_{2}\right)\left(s_{3} s_{1} s_{2} s_{3}\right)\left(s_{4} s_{3} s_{1} s_{2} s_{3} s_{4}\right)(\ldots)\left(s_{m} \ldots s_{4} s_{3} s_{1} s_{2} s_{3} s_{4} \ldots s_{m}\right),
\end{aligned}
$$

where $s_{1}, \ldots, s_{m+1} \in W$ are simple reflections. By using (3.11) and by computing the right action of $w_{m+1} \in W_{P_{m+1}}$ on a weight $\omega_{1}$, we can derive the following formula inductively.

$$
\widetilde{w}_{m+1}= \begin{cases}\left(s_{1}\right)\left(s_{3} s_{2}\right)\left(s_{4} s_{3} s_{1}\right)(\ldots)\left(s_{m} \ldots s_{4} s_{3} s_{1}\right) & \text { for even } m, \\ \left(s_{1}\right)\left(s_{3} s_{2}\right)\left(s_{4} s_{3} s_{1}\right)(\ldots)\left(s_{m} \ldots s_{4} s_{3} s_{2}\right) & \text { for odd } m .\end{cases}
$$

Similarly, we can also check by an induction that the right action of $w_{1} \widetilde{w}_{m+1} \in W$ on a weight $\omega_{2}$ is the same as that of

$$
\left(s_{2} s_{3} s_{4} \ldots s_{m+1}\right) \widetilde{w}_{m+1} \in W^{P_{2}}
$$

which is nothing but the longest element $w^{P_{2}} \in W^{P_{2}}$ as the lengths coincide.

Consider the embedding $F_{1} \subset G\left(m, V_{\text {nat }}\right)$ in Remark 2.3, For each maximal isotropic subspace $V \subset V_{\text {nat }}$, the inner product $\langle-,-\rangle$ gives a canonical linear isormorphism $V_{\text {nat }} / V \simeq V^{\vee}$. Hence we have a short exact sequence

$$
\left.\left.0 \rightarrow \mathcal{S}\right|_{F_{1}} \rightarrow V_{\text {nat }} \otimes \mathcal{O}_{F_{1}} \rightarrow \mathcal{S}^{\vee}\right|_{F_{1}} \rightarrow 0
$$

on $F_{1}$, where $\mathcal{S}$ is the universal subbundle on $G\left(m, V_{\text {nat }}\right)$. Recall that $\left.\mathcal{S}^{\vee}\right|_{F_{1}}$ is the equivariant vector bundle associated with the irreducible representation of $P_{1}$ with the highest weight $\omega_{m}$, so that $H^{0}\left(\left.S^{\vee}\right|_{F_{1}}\right) \simeq V_{\text {nat }}^{\vee}=V_{\text {nat }}$ as $\operatorname{Spin}(2 m)$-modules.

From now, we consider only the case $m=4$. By the triality for the Dynkin diagram of type $D_{4}$, we have another short exact sequence on $F_{1}$,

$$
0 \rightarrow \mathcal{E}^{\vee} \rightarrow V_{2}^{\vee} \otimes \mathcal{O}_{F_{1}} \rightarrow \mathcal{E} \rightarrow 0 .
$$

Consider the morphism

$$
\mu: \mathbb{P}:=\mathbb{P}_{F_{1}}\left(\mathcal{O}_{F_{1}}(-1) \oplus \mathcal{E}^{\vee}\right) \rightarrow \mathbb{P}\left(V_{1} \oplus V_{2}^{\vee}\right)
$$

defined by the natural inclusion

$$
\mathcal{O}_{F_{1}}(-1) \oplus \mathcal{E}^{\vee} \subset\left(V_{1} \oplus V_{2}^{\vee}\right) \otimes \mathcal{O}_{F_{1}}
$$

Let $\Sigma:=\mu(\mathbb{P}) \subset \mathbb{P}\left(V_{1} \oplus V_{2}^{\vee}\right)$ be the image of $\mu$ with the reduced structure.

Lemma 3.3. There exists an isomorphism $\mathrm{H}_{2} \stackrel{\sim}{\rightarrow} \Sigma$ which fits in the following commutative diagram.

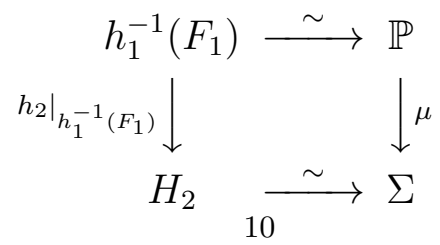


Proof. We write $\mathbb{P}^{\prime}:=h_{1}^{-1}\left(F_{1}\right)$ for simplicity. Since $\operatorname{dim} \mathbb{P}=\operatorname{dim} H_{2}$, it follows from [CR13, Lemma 3.13] that the morphism $\left.h_{2}\right|_{\mathbb{P}^{\prime}}$ is a birational morphism. Since $H_{2}$ is smooth and hence normal, it follows that $\left(h_{2} \mid \mathbb{P}^{\prime}\right)_{*} \mathcal{O}_{\mathbb{P}^{\prime}} \simeq \mathcal{O}_{H_{2}}$, so that $H^{0}\left(\mathbb{P}^{\prime},\left(h_{2} \mid \mathbb{P}^{\prime}\right)^{*} \mathcal{O}_{H_{2}}(1)\right) \simeq$ $H^{0}\left(H_{2}, \mathcal{O}_{H_{2}}(1)\right)$. Therefore, the composition of the morphism $\left.h_{2}\right|_{\mathbb{P}^{\prime}}$ with the embedding $H_{2} \hookrightarrow \mathbb{P} H^{0}\left(H_{2}, \mathcal{O}_{H_{2}}(1)\right)^{\vee}$ coincides with the morphism defined by the complete linear system associated to the line bundle $\left(\left.h_{2}\right|_{\mathbb{P}^{\prime}}\right)^{*} \mathcal{O}_{H_{2}}(1)$.

Also, the isomorphism $H^{0}\left(\mathbb{P}, \mathcal{O}_{\pi}(1)\right) \simeq V_{1}^{\vee} \oplus V_{2}$ implies that the morphism $\mu$ is associated to the complete linear system of the line bundle $\mathcal{O}_{\pi}(1)$. Therefore it is enough to show that the line bundle $\left(\left.h_{2}\right|_{\mathbb{P}^{\prime}}\right)^{*} \mathcal{O}_{H_{2}}(1)$ on $\mathbb{P}^{\prime}$ corresponds to $\mathcal{O}_{\pi}(1)$ on $\mathbb{P}$ under the isomorphism $\mathbb{P}^{\prime} \simeq \mathbb{P}$. Note, by the commutative diagram (3.3), this is equivalent to the isomorphism $\mathcal{O}_{\left.h_{1}\right|_{\mathbb{P}}}(1) \simeq\left(\left.h_{2}\right|_{\mathbb{P}^{\prime}}\right)^{*} \mathcal{O}_{H_{2}}(1)$.

To see this, consider the projective bundle $h_{1}: H_{1,2}=\mathbb{P}_{H_{1}}(\mathcal{G}) \rightarrow H_{1}$. The isomorphism $\mathcal{G}^{\vee} \simeq h_{1 *} h_{2}^{*} \mathcal{O}_{H_{2}}(1)$ implies that $\mathcal{O}_{h_{1}}(1) \simeq h_{2}^{*} \mathcal{O}_{H_{2}}(1)$. On the other hand, since the projective bundle $\left.h_{1}\right|_{\mathbb{P}^{\prime}}: \mathbb{P}^{\prime}=\mathbb{P}_{F_{1}}\left(\left.\mathcal{G}\right|_{F_{1}}\right) \rightarrow F_{1}$ is obtained as the base change of $h_{1}$ by $F_{1} \hookrightarrow H_{1}$, we obtain the sequence of isomorphisms $\left.\left.\mathcal{O}_{\left.h_{1}\right|_{\mathbb{P}^{\prime}}}(1) \simeq \mathcal{O}_{h_{1}}(1)\right|_{\mathbb{P}^{\prime}} \simeq h_{2}^{*} \mathcal{O}_{H_{2}}(1)\right|_{\mathbb{P}^{\prime}}=$ $\left(\left.h_{2}\right|_{\mathbb{P}^{\prime}}\right)^{*} \mathcal{O}_{H_{2}}(1)$.

Now we restrict ourselves to the case $m=4$, where we have the following diagram:

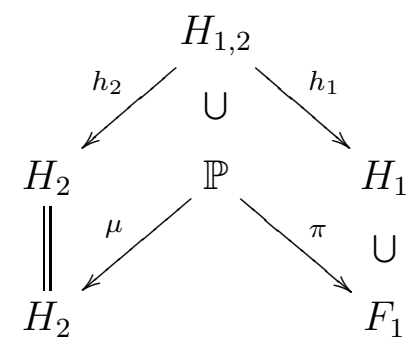

Let $\sigma \in H^{0}\left(F_{1}, \mathcal{E}(1)\right)$ be the image of a linear map $\widetilde{\sigma}: V_{1} \rightarrow V_{2}^{\vee}$ by the map

$$
V_{1}^{\vee} \otimes V_{2}^{\vee} \simeq H^{0}\left(\mathcal{O}_{F_{1}}(1)\right) \otimes H^{0}(\mathcal{E}) \rightarrow H^{0}(\mathcal{E}(1)) \text {. }
$$

The map (3.20) is surjective, since it is a non-zero $\operatorname{Spin}(8)$-equivariant map and $H^{0}(\mathcal{E}(1)) \simeq$ $V_{(1,1)}^{\vee}$ is an irreducible representation of $\operatorname{Spin}(8)$. In particular, $\sigma$ is general if so is $\widetilde{\sigma}$. On the other hand, the map $\widetilde{\sigma}$ induces a linear embedding

$$
\mathbb{P}\left(\Gamma_{\widetilde{\sigma}}\right): \mathbb{P}\left(V_{1}\right) \hookrightarrow \mathbb{P}\left(V_{1} \oplus V_{2}^{\vee}\right), \quad[p] \mapsto[(p, \widetilde{\sigma}(p))],
$$

whose image will be denoted by $L_{\widetilde{\sigma}} . \subset \mathbb{P}\left(V_{1} \oplus V_{2}^{\vee}\right)$. Conversely, if a 7-dimensional linear subvariety $L \subset \mathbb{P}\left(V_{1} \oplus V_{2}^{\vee}\right)$ satisfies $L \cap \mathbb{P}\left(V_{2}^{\vee}\right)=\emptyset$, then there exists an element $\tilde{\sigma} \in$ $V_{1}^{\vee} \otimes V_{2}^{\vee}$ such that $L=L_{\widetilde{\sigma}}$. It means that $L_{\widetilde{\sigma}}$ is general if so is $\widetilde{\sigma}$. Thus a general $\sigma \in H^{0}(\mathcal{E}(1))$ corresponds to a general $L_{\widetilde{\sigma}} \subset \mathbb{P}\left(V_{1} \oplus V_{2}^{\vee}\right)$.

Proposition 3.4 below is an adaptation of [IIM19, Proposition 4.1] to the present situation. The proof is identical, and hence omitted.

Proposition 3.4. The zero locus $Z(\sigma) \subset F_{1} \subset \mathbb{P}\left(V_{1}\right)$ of $\sigma \in H^{0}(\mathcal{E}(1))$ and the linear section $H_{2} \cap L_{\widetilde{\sigma}} \subset L_{\widetilde{\sigma}} \simeq \mathbb{P}\left(V_{1}\right)$ are projectively equivalent.

One concludes that a general linear section of $H_{2}=\mathrm{OG}(5,10)$ is projectively equivalent (and hence isomorphic) to the zero of a general section of $\mathcal{E}(1)$ on $F_{1}=\mathrm{OG}(4,8)$, which is nothing but $X$ in Section 2 for $m=4$.

\section{Projective Duality And Derived equivalence}

The orthogonal Grassmannian $\mathrm{OG}(5,10)$ of dimension 10 can be embedded into the projectivization $\mathbb{P}$ of 16 -dimensional half spinor representation of $\operatorname{Spin}(10)$. The projective 
dual variety of $\mathrm{OG}(5,10)$ in the dual projective space $\check{\mathbb{P}}$ is also isomorphic to $\mathrm{OG}(5,10)$. It is known in [Muk88, Theorem 0.3] that a generic K3 surface of degree 12 can be described as the intersection $\mathrm{OG}(5,10) \cap L$ with a linear subspace $L \subset \mathbb{P}^{15}$ of codimension 8 , which is unique up to the action of $\operatorname{Spin}(10)$.

Consider a pair of $\mathrm{K} 3$ surface $(X, Y)$ which is constructed as in (2.9) for $m=4$, and realize $X$ as $\mathrm{OG}(5,10) \cap L$. As explained in [Muk99, Example 1.3], the intersection

$$
\check{X}:=\mathrm{OG}(5,10) \cap L^{\perp} \in \check{\mathbb{P}}^{15}
$$

of the dual $\mathrm{OG}(5,10)$ with $L^{\perp}$ in the dual projective space is the moduli space

$$
\check{X} \simeq \mathcal{M}_{X}\left(2, \mathcal{O}_{X}(1), 3\right),
$$

where $\mathcal{M}_{X}(r, \ell, t)$ is the moduli space of stable sheaves $E$ on $X$ satisfying

$$
\operatorname{rank} E=r, c_{1}(E)=\ell, \chi(E)=r+t .
$$

$(X, \check{X})$ give Fourier-Mukai partners, which are not isomorphic if $X$ has Picard number one by [HLOY03, Theorem 2.1] (see also its proof).

Proposition 4.1. One has an isomorphism $\check{X} \simeq Y$.

Proof. We use the notation introduced in Section 3. A general element $\tilde{\sigma} \in V_{1}^{\vee} \otimes V_{2}^{\vee}$ defines the linear subspaces

$$
L_{1}:=\operatorname{Im}\left(\left(\operatorname{id}_{V_{1}}, \tilde{\sigma}\right): \mathbb{P}\left(V_{1}\right) \rightarrow \mathbb{P}\left(V_{1} \oplus V_{2}^{\vee}\right)\right)
$$

and

$$
L_{2}:=\operatorname{Im}\left(\left(-\widetilde{\sigma}, \operatorname{id}_{V_{2}}\right): \mathbb{P}\left(V_{2}\right) \rightarrow \mathbb{P}\left(V_{1}^{\vee} \oplus V_{2}\right)\right),
$$

in the dual projective spaces, which are mutually orthogonal;

$$
L_{1}=\left(L_{2}\right)^{\perp}, \quad L_{2}=\left(L_{1}\right)^{\perp} .
$$

Proposition 3.4 shows

$$
X=L_{1} \cap H_{2} \subset F_{1}, \quad Y=L_{2} \cap H_{1} \subset F_{2},
$$

which immediately implies Proposition 4.1 .

Theorem 1.3 follows from Corollary 2.2 for $m=4$, the last line of Section 3 , and [Muk99, Example 1.3].

Remark 4.2. Let $\mathbb{O}$ be the complexified Cayley octonion algebra. Consider the projective space of null-square imaginary octonions

$$
Q:=\left\{\left[\sum_{i=0}^{7} x_{i} \mathbf{e}_{i}\right] \in \mathbb{P}(\mathbb{O}) \mid x_{0}=\sum_{i=1}^{7} x_{i}^{2}=0\right\},
$$

and set $Q_{1}=Q_{2}=Q$ with coordinates $x$ and $y$, respectively. Together with

$$
Q_{1,2}:=\left\{(x, y) \in Q_{1} \times Q_{2} \mid x \cdot y=0 \in \mathbb{O}\right\}
$$

they form the following diagram, where the projections $q_{1}$ and $q_{2}$ are both $\mathbb{P}^{2}$-bundles.

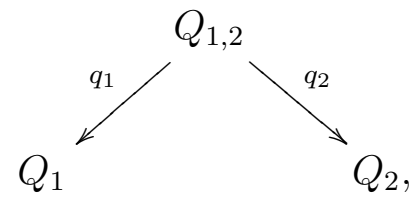


Let $s^{\prime}$ be a general section of $H^{0}\left(\mathcal{O}_{Q_{1,2}}(1,1)\right)$ and set

$$
\begin{gathered}
D^{\prime}:=Z\left(s^{\prime}\right) \subset Q_{1,2}, \\
X^{\prime}:=Z\left(q_{1 *} s^{\prime}\right) \subset Q_{1}, \\
Y^{\prime}:=Z\left(q_{2 *} s^{\prime}\right) \subset Q_{2} .
\end{gathered}
$$

Then both $X^{\prime}$ and $Y^{\prime}$ are K3 surfaces of degree 12, and the same reasoning as Corollary 2.2 gives the equality

$$
\left(\left[X^{\prime}\right]-\left[Y^{\prime}\right]\right) \mathbb{L}^{2}=0
$$

in the Grothendieck ring of varieties. In fact, the pairs $\left(X^{\prime}, Y^{\prime}\right)$ are degenerations of the pairs $(X, Y)$ discussed above. It is an interesting problem to see if $X^{\prime}$ and $Y^{\prime}$ are not isomorphic, and characterize K3 surfaces which can be obtained in this way.

\section{K3 SURFACES IN $\mathrm{G}(2,6)$}

Let $W$ be a vector space of dimension 6. The Pfaffian hypersurface $\operatorname{Pf}(W) \subset \mathbb{P}\left(\bigwedge^{2} W^{\vee}\right)$ consists of skew bilinear forms on $W$ whose rank is strictly smaller than 6 . A general linear subspace $L \subset \mathbb{P}\left(\bigwedge^{2} W^{\vee}\right)$ of dimension 5 determines a cubic 4-fold

$$
X:=\operatorname{Pf}(W) \cap L
$$

and a K3 surface

$$
Y:=\mathrm{G}(2, W) \cap L^{\perp} .
$$

It is known by [Kuz, Theorem 2] that there is a fully faithful functor $\iota: D(Y) \rightarrow D(X)$ and a semiorthogonal decomposition

$$
D(X)=\left\langle\mathcal{O}_{X}, \mathcal{O}_{X}(1), \mathcal{O}_{X}(2), \iota D(Y)\right\rangle
$$

Fix a linear subspace $U \subset W$ of dimension 5 . The rational map

$$
\varphi: \operatorname{Pf}(W) \rightarrow \mathbb{P}(U)
$$

induced by the composition of the wedge square $(-)^{\wedge 2}: \bigwedge^{2} W^{\vee} \rightarrow \bigwedge^{4} W^{\vee}$, the projection $\bigwedge^{4} W^{\vee} \rightarrow \bigwedge^{4} U^{\vee}$, and the (canonical up to scalar) isomorphism $\bigwedge^{4} U^{\vee} \stackrel{\sim}{\rightarrow} U$ sends $x \in \bigwedge^{2} W^{\vee} \subset \operatorname{Hom}\left(W, W^{\vee}\right)$ to ker $x \cap U \subset U$. It is defined by a linear subsystem of $|2 H|$, where $H$ is the hyperplane section of $\operatorname{Pf}(W)$. The base locus of this linear subsystem is the union of

$$
B_{1}:=\{x \in \operatorname{Pf}(W) \mid \operatorname{ker} x \subset U\}
$$

and

$$
B_{2}:=\{x \in \operatorname{Pf}(W) \mid \operatorname{dim} \operatorname{ker} x=4\} .
$$

The inclusion $U \subset W$ induces a linear projection $\pi_{U}: \mathbb{P}\left(\bigwedge^{2} W^{\vee}\right) \rightarrow \mathbb{P}\left(\bigwedge^{2} U^{\vee}\right)$, and $B_{1}$ is the closure of the inverse image of

$$
\mathrm{G}(2, U)=\left\{x \in \mathbb{P}\left(\bigwedge^{2} U^{\vee}\right) \mid \operatorname{dim} \operatorname{ker} x=3\right\} \subset \mathbb{P}\left(\bigwedge^{2} U^{\vee}\right)
$$

by this rational map. If $L$ is general, then $B_{2} \cap L$ is empty, and $S:=B_{1} \cap L$ projects isomorphically by $\pi_{U}$ to the section $\mathrm{G}(2, U) \cap \pi_{U}(L) \subset \mathbb{P}\left(\bigwedge^{2} U^{\vee}\right)$ of $\mathrm{G}(2, U)$ by the linear subspace $\pi_{U}(L)$ of dimension 5 , which is a del Pezzo surface of degree 5 embedded anti-canonically into $\pi_{U}(L) \simeq L$. Let $\widetilde{X}:=\mathrm{Bl}_{S} X$ be the blow-up of $X$ along $S$, whose exceptional divisor will be denoted by $E$. It is known by [Tre84, Proposition 2] that 
- the linear system $|2 \widetilde{H}-E|$ gives a birational morphism $\widetilde{\varphi}: \widetilde{X} \rightarrow \mathbb{P}(U)$, where $\widetilde{H}$ is the total transform of the hyperplane section of $X$, and

- the exceptional locus $F \subset \widetilde{X}$ of $\widetilde{\varphi}$ is a smooth divisor, whose image $G \subset \mathbb{P}^{4}$ is isomorphic to the 5-point blow-up of $Y$.

The proof of [Tre84, Proposition 2], which is based on [Dan80, Theorem 1], actually shows that the morphism $\widetilde{\varphi}$ is the blow-up of $\mathbb{P}^{4}$ along $G$. Hence one has

$$
\begin{aligned}
{[\tilde{X}] } & =[X]+\mathbb{L}[S] \\
& =[X]+\mathbb{L}\left(1+\mathbb{L}+\mathbb{L}^{2}+4 \mathbb{L}\right)
\end{aligned}
$$

and

$$
\begin{aligned}
{[\tilde{X}] } & =\left[\mathbb{P}^{4}\right]+\mathbb{L}[G] \\
& =1+\mathbb{L}+\mathbb{L}^{2}+\mathbb{L}^{3}+\mathbb{L}^{4}+\mathbb{L}([Y]+5 \mathbb{L})
\end{aligned}
$$

so that

$$
[X]=1+\mathbb{L}^{2}+\mathbb{L}^{4}+\mathbb{L}[Y] .
$$

This is compatible with the SOD (5.3) under the motivic measure (1.15), in the sense that the terms in the right hand side of (5.8) are sent to the summands of (5.3).

Remark 5.1. We learned from Genki Ouchi that he independently obtained the formula (5.12) multiplied by $\mathbb{L}^{5}(\mathbb{L}-1)^{2}(\mathbb{L}+1)$ using an argument similar to Bor18. See [KS, Section 2.6] for similar results for other types of cubic fourfolds.

\section{Abelian varieties}

We prove Theorem [1.5 in this section. First recall the following definition.

Definition 6.1. The localized Grothendieck ring of varieties $K_{0}(\mathcal{V}$ ar $/ \mathbf{k})\left[\mathbb{L}^{-1}\right]$ is the localization of the Grothendieck ring of varieties $K_{0}(\mathcal{V}$ ar $/ \mathbf{k})$ by the class $\mathbb{L}$ of the affine line. For each integer $i \in \mathbb{Z}$, let $\operatorname{Fil}_{i} \subset K_{0}(\mathcal{V}$ ar $/ \mathbf{k})\left[\mathbb{L}^{-1}\right]$ be the abelian subgroup spanned by the elements of the form $[X] \cdot \mathbb{L}^{-m}$, where $m \in \mathbb{Z}$ is an integer and $X$ is a variety such that $\operatorname{dim} X-m \leq i$. The subgroups $\left(\mathrm{Fil}_{i}\right)_{i \in \mathbb{Z}}$ form an ascending filtration of $K_{0}(\mathcal{V}$ ar $/ \mathbf{k})\left[\mathbb{L}^{-1}\right]$ satisfying $\mathrm{Fil}_{i} \cdot \mathrm{Fil}_{j} \subset \mathrm{Fil}_{i+j}$. The completed Grothendieck ring of varieties is the completion with respect to this filtration:

$$
K_{0}(\mathcal{V} \text { ar } / \mathbf{k})\left[\mathbb{L}^{-1}\right] \rightarrow \lim _{i \in \mathbb{Z}} \frac{K_{0}(\mathcal{V a r} / \mathbf{k})\left[\mathbb{L}^{-1}\right]}{\operatorname{Fil}_{i}}=: \widehat{K}_{0}(\mathcal{V} \text { ar } / \mathbf{k})
$$

Let $A_{\mathbf{k}}$ be the group completion of the commutative monoid of isomorphism classes of algebraic group schemes over $\mathbf{k}$ whose connected components are abelian varieties and whose group of geometric connected components is a finitely generated group, where the binary operation + of the monoid is defined by the direct sum; $[A]+[B]:=[A \oplus B]$. When $\mathbf{k}=\mathbb{C}$, by using the Bittner presentation of the Grothendieck ring [Bit04] (which in turn is based on resolution of singularities [Hir64] and weak factorization [AKMW02]), Ekedahl proved the following:

Theorem 6.2 ([Eke09, Theorem 3.4], cf. also [Cau16, Appendix A]). There is a homomorphism of abelian groups

$$
\operatorname{Pic}_{\mathbb{C}}: \widehat{K}_{0}(\mathcal{V} \text { ar } / \mathbb{C}) \rightarrow A_{\mathbb{C}}
$$

sending the class $[X]$ of a smooth proper variety $X$ to the class $[\operatorname{Pic}(X)]=\left[\operatorname{Pic}^{0}(X)\right]+$ $[\mathrm{NS}(X)]$. 
Ekedahl also proved the following:

Proposition 6.3 ([Eke09, Proposition 3.6]). Assume that $A, B$ and $C$ are abelian varieties over $\mathbb{C}$ satisfying $A \oplus C \simeq B \oplus C$. Then $\operatorname{Hom}(A, B)$ is a locally free right module of rank 1 over $R:=\operatorname{End}(A)$ and the natural morphism of abelian varieties $\operatorname{Hom}(A, B) \otimes_{R} A \rightarrow B$ is an isomorphism.

The proof of [Eke09, Proposition 3.6] is based on the Tate's isogeny theorem for abelian varieties over a field which is finitely generated over its prime field. This is first shown in [Fal83] over number fields, and is generalized later (see [FW84, Chapter VI §3 Theorem 1 b)]. See also [FW84, Chapter IV $\S 1$ Corollary 1.2] and its proof).

Corollary 6.4. Let $X$ and $Y$ be smooth projective varieties over $\mathbb{C}$ such that $\operatorname{End}\left(\operatorname{Pic}^{0}(X)\right) \simeq$ $\mathbb{Z}$. If $[X]=[Y]$ holds in $\widehat{K}_{0}(\mathcal{V}$ ar $/ \mathbb{C})$, then $\operatorname{Pic}^{0}(X)$ is isomorphic to $\operatorname{Pic}^{0}(Y)$.

Proof. Theorem 6.2 gives the equality $[\operatorname{Pic}(X)]=[\operatorname{Pic}(Y)]$ in the group $A_{\mathbb{C}}$. This is equivalent to saying that there exists a group scheme $G$ whose neutral component is an abelian variety and the group of components is a finitely generated abelian group such that

$$
\operatorname{Pic}(X) \times G \simeq \operatorname{Pic}(Y) \times G .
$$

By taking the neutral components, we obtain the isomorphism

$$
\operatorname{Pic}^{0}(X) \times G^{0} \simeq \operatorname{Pic}^{0}(Y) \times G^{0} .
$$

Now Proposition 6.3 gives an isomorphism $\operatorname{Pic}^{0}(X) \simeq \operatorname{Pic}^{0}(Y)$, since a locally free $\mathbb{Z}$ module of rank 1 is unique up to isomorphism.

Remark 6.5. A ring $R$ is said to have the cancellation property if an isomorphism of finitely generated one-sided modules $A \oplus C \simeq B \oplus C$ over $R$ implies $A \simeq B$ whenever $C$ is a projective module. In Corollary 6.4, it suffices to assume that End $\left(\operatorname{Pic}^{0}(X)\right)$ is a hereditary ring with the cancellation property.

Lemma 6.6. For any integer $g \geq 2$, there exists an abelian $g$-fold $A$ such that End $(A)=$ $\mathbb{Z}$ and $A \not \widehat{A}:=\operatorname{Pic}^{0}(A)$.

Proof. The authors learned the following construction of $A$ from the answer by Bjorn Poonen to a question in MathOverflow [Poo]. For the sake of completeness, here we give an explanation with details (with a minor change).

Let $J$ be the Jacobian of a very general smooth projective curve of genus $g$. As explained in [Dol16, Lecture 15], by applying [ACGH85, p. 359 Lemma] for the case $d=2$ and [BL04, Theorem 11.5.1] we obtain $\operatorname{End}(J)=\mathbb{Z}$. Note also that $J$ is isomorphic to its dual, since it is the Jacobian of a curve and hence is a principally polarized abelian variety. Let $G \subset J$ be a finite subgroup whose order $n$ is not a $g$-th power of an integer. Then $A:=J / G$ is never isomorphic to its dual, since otherwise the composition

$$
J \stackrel{q}{\rightarrow} A \stackrel{\sim}{\rightarrow} \widehat{A} \stackrel{\widehat{q}}{\rightarrow} \widehat{J} \stackrel{\sim}{\rightarrow} J
$$

gives an endomorphism of $J$ of degree $n^{2}$, contradicting End $(J)=\mathbb{Z}$ and the choice of $n$. The isogeny $q: J \rightarrow A$ induces the isomorphism $\operatorname{End}(A) \otimes \mathbb{Q} \simeq \operatorname{End}(J) \otimes \mathbb{Q}=\mathbb{Q}$, and hence one has $\operatorname{End}(A)=\mathbb{Z}$.

Theorem 1.5 is an immediate consequence of Corollary 6.4 and Lemma 6.6. Note that the existence of one example in Lemma 6.6 implies that a very general abelian variety also is an example. 
We can also construct another class of examples of D-equivalent varieties whose classes are distinct in $\widehat{K}_{0}(\mathcal{V}$ ar $/ \mathbb{C})$.

Corollary 6.7. Let $A$ be a complex abelian surface such that $A \neq \widehat{A}$ and $\operatorname{End}(A)=\mathbb{Z}$. Take a natural number $n \geq 1$ and consider the Hilbert schemes of n-points $A^{[n]}$ and $\widehat{A}^{[n]}$. Then the pair $\left(A^{[n]}, \widehat{A}^{[n]}\right)$ gives a negative answer to Problem 1.2.

Proof. By [Plo07, Proposition 8], the derived equivalence between $A$ and $\widehat{A}$ induces a derived equivalence between $A^{[n]}$ and $\widehat{A}^{[n]}$. On the other hand, the summation maps $s: A^{[n]} \rightarrow A$ and $\widehat{s}: \widehat{A}^{[n]} \rightarrow \widehat{A}$ are the Albanese maps of $A^{[n]}$ and $\widehat{A}^{[n]}$, respectively. Hence it follows from Corollary 6.4 that $\left[A^{[n]}\right] \neq\left[\widehat{A}^{[n]}\right] \in \widehat{K}_{0}(\mathcal{V}$ ar $/ \mathbb{C})$.

\section{VARiations of Problem 1.2}

Now that we found conterexamples to Problem 1.2, we discuss its possible modifications in this section. The guiding principle is to weaken the assertion of Problem 1.2 without losing meaningful implications such as the D-invariance of the Hodge numbers and the number of rational points over finite fields.

Before we discuss the modifications, we point out that this problem is also interesting from the point of view of the mirror symmetry. A pair $(X, \tilde{X})$ of Calabi-Yau $n$-folds is said to be a topological mirror pair if

$$
h^{p, q}(X)=h^{n-p, q}(\check{X})
$$

for $0 \leq p, q \leq n$, so that if $(X, Y)$ and $(\check{X}, \check{Y})$ are Fourier-Mukai pairs, and $(X, \check{X})$ and $(Y, \check{Y})$ are topological mirror pairs, then (7.1) together with (1.1) implies

$$
\begin{aligned}
\sum_{p+q=i} h^{p, q}(X) & =\sum_{p+q=i} h^{n-p, q}(\check{X}) \\
& =\sum_{p+q=i} h^{n-p, q}(\check{Y}) \\
& =\sum_{p+q=i} h^{p, q}(Y)
\end{aligned}
$$

for $0 \leq i \leq 2 n$. In other words, mirror symmetry exchanges the horizontal (or Hodge filtration) direction and the vertical (or weight) direction in the Hodge diamond, so that if the mirrors of Fourier-Mukai partners are Fourier-Mukai partners, then not only the vertical sums but also the horizontal sums will be preserved. Moreover, if $(X, Y)$ is a Fourier-Mukai pair, and $(X, Z)$ and $(Y, Z)$ are topological mirror pairs, then one has

$$
h^{p, q}(X)=h^{n-p, q}(Z)=h^{p, q}(Y) .
$$

This is the case, for example, for the Pfaffian-Grassmannian pair of Calabi-Yau 3-folds $\mathrm{R} \varnothing \mathrm{d} 00$ mentioned in Introduction.

We also note that the coincidence of the Hasse-Weil zeta functions is stronger than the coincidence of the Hodge numbers in the following sense.

Proposition 7.1. Assume in dimension d and over finite fields that the D-equivalence implies the coincidence of the Hasse-Weil zeta functions. Then it follows in the same dimension $d$ and over $\mathbb{C}$ that the D-equivalence implies the coincidence of the Hodge numbers.

Proof. 
Step 1. We reduce the proof to the case where everything is defined over a number field $K$. Choose projective embeddings of $X$ and $Y$ into a projective space $\mathbb{P}_{\mathbb{C}}$, so that they are defined by systems of homogeneous polynomials with coefficients in $\mathbb{C}$. We choose such embeddings that $H^{1}\left(\mathbb{P}_{\mathbb{C}}, I_{X / \mathbb{P}_{\mathbb{C}}}(i)\right)=0=H^{1}\left(\mathbb{P}_{\mathbb{C}}, I_{Y / \mathbb{P}_{\mathbb{C}}}(i)\right)$ holds for all $i>0$, so that $H^{0}\left(\mathbb{P}_{\mathbb{C}}, \mathcal{O}_{\mathbb{P}_{\mathbb{C}}}(i)\right) \rightarrow H^{0}\left(X, \mathcal{O}_{X}(i)\right)$ is surjective for all $i \geq 0$ (resp. for $Y$ ). Next we replace the Fourier-Mukai kernel of the D-equivalence between $X$ and $Y$ with a complex $E$ consiting of direct sums of line bundles of the form $\mathcal{O}_{X}(i) \otimes \mathcal{O}_{Y}(i)$ for some integer $i$. By the surjectivity we just mentioned, the differentials of the perfect complex are represented by matrices whose terms are polynomials over $\mathbb{C}$. Thus we see that there exists a subring $R \subset \mathbb{C}$ of finite type over $\mathbb{Q}$ such that

- $X, Y$ are the base changes by $Q(R) \subset \mathbb{C}$ of the fibers over the generic point Spec $Q(R) \rightarrow \operatorname{Spec} R$ of the schemes $\mathcal{X}$ and $\mathcal{Y}$ which are defined in the projective space $\mathbb{P}_{R}$ over $R$ by a system of homogeneous polynomials with coefficients in $R$, and

- the Fourier-Mukai kernel $E$ is the base change of the restriction to the generic fiber of a perfect complex $\mathcal{E}$ on $\mathbb{P}_{R} \times_{R} \mathbb{P}_{R}$.

Now consider the variety $T=\operatorname{Spec} R$ in characteristic zero. Since $X$ and $Y$ are smooth and projective over the generic point of $T$, there exists an open dense subset $U \subset T$ over which $\mathcal{X}$ and $\mathcal{Y}$ are smooth and projective. Since the Hodge numbers are the same for all the fibers of a smooth projective morphism between varieties in characteristic zero, we can compare the Hodge numbers of $X$ and $Y$ on the fibers of any closed point of $U$ (see the similar argument in the proof of [Ito03, Proposition 5.1]).

Take the the right adjoint kernel $\mathcal{E}_{R}=\mathcal{E}^{\vee} \otimes p_{\mathcal{X}}^{*} \omega_{\mathcal{X} / R}[\operatorname{dim} X]$ of $\mathcal{E}$ (see, say, Huy06, Definition 5.7]). Consider the adjunction unit map

$$
\mu: \mathcal{O}_{\Delta_{\mathcal{X} / R}} \rightarrow \mathcal{E}_{R} * \mathcal{E}
$$

where $*$ denotes the convolution of kernels $p_{\mathcal{X}, \mathcal{X} *}\left(p_{\mathcal{Y}, \mathcal{X}}^{*} \mathcal{E}_{R} \otimes p_{\mathcal{X}, \mathcal{Y}}^{*} \mathcal{E}\right)$, so that

$$
\Phi_{\mathcal{E}_{R} * \mathcal{E}}^{\mathcal{X} \rightarrow \mathcal{X}}=\Phi_{\mathcal{E}_{R}}^{\mathcal{Y} \rightarrow \mathcal{X}} \circ \Phi_{\mathcal{E}}^{\mathcal{X} \rightarrow \mathcal{Y}}
$$

Since $\Phi_{E}^{X \rightarrow Y}$ is fully faithful, it follows that the support of the cone of $\mu$ does not intersect the generic fiber $\mathcal{X}_{Q(R)}$. Since $\mathcal{X}$ is proper over $T$, by removing the image of the support of the cone of $\mu$ if necessary, we may assume that $\mu$ is an isomorphism over $U$. The flatness of $\mathcal{Y}$ over $U$ implies that $p_{\mathcal{X}, \mathcal{X}_{*}}$ commutes with restriction over any point $u \in U$, so that one can easily verify $\left.\left.\left.\left.\left(\mathcal{E}_{R} * \mathcal{E}\right)\right|_{u} \simeq \mathcal{E}_{R}\right|_{u} * \mathcal{E}\right|_{u} \simeq\left(\left.\mathcal{E}\right|_{u}\right)_{R} * \mathcal{E}\right|_{u}$. Thus we see that $\Phi_{\left.\mathcal{E}\right|_{u}}$ is fully faithful for any $u \in U$. Combined with similar arguments for the adjunction counit map, one can deduce that $\Phi_{\left.\mathcal{E}\right|_{u}}$ is an equivalence for any $u \in U$ after removing some non-dense closed subset from $U$ if necessary. Therefore we can take the number field $K$ to be the residue field of any closed point of $U$.

Step 2. Once everything is defined over a number field $K$, then $X, Y$ extend to projective schemes over the ring of integers $\mathcal{O}_{K} \subset K$ and the Fourier-Mukai kernel $E$ extend to a perfect complex on their fiber product over $\mathcal{O}_{K}$ (recall that we may assume that $E$ is a perfect complex whose terms are direct sums of line bundles of the form $\mathcal{O}_{X}(i) \otimes \mathcal{O}_{Y}(i)$ and the differentials are matrices whose entries are polynomials with coefficients in $K$ ). Moreover, by similar arguments as above, one can find an open dense subset $V \subset \operatorname{Spec} \mathcal{O}_{K}$ over which $X$ and $Y$ extend to smooth schemes and also the Fourier-Mukai kernel $E$ yields D-equivalence between any pair of fibers. Now, if one assumes the conjecture that Dequivalence implies the coincidence of Hasse-Weil zeta functions over finite fields, one can apply it to the reductions of $X$ and $Y$ to the closed points of $V$. This then would 
imply the coincidence of the Hodge numbers in characteristic zero by [Ito03, Proposition $1.2]$.

Now we consider the possible modifications of Problem 1.2. In the study of arithmetic and geometry of abelian varieties, it is often convenient to work with the category of abelian varieties up to isogeny. Let $K_{0}^{\prime}(\mathcal{V}$ ar $/ \mathbf{k})$ be the quotient of $K_{0}(\mathcal{V}$ ar $/ \mathbf{k})$ by the ideal generated by $[A]-[B]$, where $A$ and $B$ are isogenous abelian varieties. The counting measure factors through $K_{0}^{\prime}\left(\mathcal{V}\right.$ ar $\left./ \mathbb{F}_{q}\right)\left[\mathbb{L}^{-1}\right]$, and the Hodge-Deligne polynomial factors through the composition with the completion $K_{0}^{\prime}(\mathcal{V}$ ar $/ \mathbb{C})\left[\mathbb{L}^{-1}\right] \rightarrow \widehat{K}_{0}^{\prime}(\mathcal{V}$ ar $/ \mathbb{C})$ with respect to the filtration induced by that of $K_{0}(\mathcal{V}$ ar $/ \mathbf{k})$ in Definition 6.1. In view of the result by Orlov Orl02 that D-equivalent abelian varieties are isogenous, it is natural to ask the following:

Problem 7.2. Let $(X, Y)$ be a Fourier-Mukai pair. Does the equality $[X]=[Y]$ hold either in $K_{0}^{\prime}(\mathcal{V}$ ar $/ \mathbf{k})\left[\mathbb{L}^{-1}\right]$ or $\widehat{K}_{0}^{\prime}(\mathcal{V}$ ar $/ \mathbf{k})$ ?

One could instead consider the Grothendieck ring $K_{0}\left(\mathrm{CM}_{\mathbf{k}}\right)$ of the $\mathbb{Q}$-linear tensor category $\mathrm{CM}_{\mathbf{k}}$ of Chow motives with rational coefficients over $\mathbf{k}$. The Chow motive of a smooth projective variety $X$ will be denoted by $\mathfrak{h}(X)$.

Problem 7.3. Let $(X, Y)$ be a Fourier-Mukai pair. Does the equality $[\mathfrak{h}(X)]=[\mathfrak{h}(Y)]$ hold in $K_{0}\left(\mathrm{CM}_{\mathbf{k}}\right)$ ?

A conjecture of Orlov Orl05, Conjecture 1], which asserts that the effective numerical Chow motives of any Fourier-Mukai pair $(X, Y)$ are isomorphic, implies the affirmative answer to Problem [7.3. Here we note that the conjecture has been confirmed for some of the examples mentioned in the introduction, in a series of works by Laterveer Lat18b, Lat19, Lat18a.

Orlov's conjecture is partly motivated by the fact, proved in Orl05, Tab05, Kon09, Tab13, that for any Fourier-Mukai pair $(X, Y)$, the images of $\mathfrak{h}(X)$ and $\mathfrak{h}(Y)$ in the orbit category $\mathrm{CM}_{\mathbf{k}} / \mathbb{Z}^{\mathbb{Q}(1) \otimes(-)}$ of $\mathrm{CM}_{\mathbf{k}}$ with respect to the Tate twist functor $\mathbb{Q}(1) \otimes$ $(-)$ are isomorphic. This roughly means that we can recover the motive $\mathfrak{h}(X)$ of a smooth projective variety $X$ from the derived category $D(X)$ up to the information of codimensions of cycles. Problem 7.3 asks if this remaining information can also be recovered. For the case $\mathbf{k}=\mathbb{F}_{q}$ one should note that an affirmative answer to Problem 7.3 only implies the coincidence of the number of rational points modulo $q$ (see the paragraph after Problem 7.5 below), whereas an affirmative answer to Problem 7.2 implies the actual coincidence.

When $\mathbf{k}$ is of characteristic zero, there is a motivic measure

$$
\mu_{\mathrm{GS}}: K_{0}(\mathcal{V} \text { ar } / \mathbf{k})\left[\mathbb{L}^{-1}\right] \rightarrow K_{0}\left(\mathrm{CM}_{\mathbf{k}}\right)
$$

due to [?] which sends the class $[X]$ of a smooth proper variety $X$ to $[\mathfrak{h}(X)]$. Since isogenous abelian varieties have isomorphic Chow motives (cf. e.g. MNP13, Theorem 2.7.2 (c)]), it factors through a ring homomorphism $K_{0}^{\prime}(\mathcal{V}$ ar $/ \mathbf{k})\left[\mathbb{L}^{-1}\right] \rightarrow K_{0}\left(\mathrm{CM}_{\mathbf{k}}\right)$. Hence, in characteristic 0, the affirmative answer to Problem 7.2 implies that to Problem 7.3 .

Remark 7.4. The difference $[A]-[\widehat{A}] \in K_{0}(\mathcal{V}$ ar $/ \mathbb{C})\left[\mathbb{L}^{-1}\right]$ for an abelian variety $A$ as in Lemma 6.6 is a non-trivial element of the kernel of $\mu_{\mathrm{GS}}$, since $A$ and $\widehat{A}$ are isogenous.

One can also consider the category $\mathrm{NM}_{\mathbf{k}}$ of numerical motives with rational coefficients over $\mathbf{k}$. The numerical motive of a smooth projective variety $X$ will be denoted by $\mathfrak{n}(X)$. The numerical version of Problem 7.3 is the following: 
Problem 7.5. Let $(X, Y)$ be a Fourier-Mukai pair. Does the equality $[\mathfrak{n}(X)]=[\mathfrak{n}(Y)]$ hold in $K_{0}\left(\mathrm{NM}_{\mathbf{k}}\right)$ ?

The affirmative answer to Problem 7.3 implies that to Problem 7.5. Unlike the case of Chow motives, the equality $[\mathfrak{n}(X)]=[\mathfrak{n}(Y)]$ in $K_{0}\left(\mathrm{NM}_{\mathbf{k}}\right)$ is close to the equality $\mathfrak{n}(X)=\mathfrak{n}(Y)$ in $\mathrm{NM}_{\mathbf{k}}$, since $\mathrm{NM}_{\mathbf{k}}$ is semisimple abelian [Jan92. The affirmative answer to Problem 7.5 for a Fourier-Mukai pair $(X, Y)$ over the finite field $\mathbb{F}_{q}$ implies the equality of the number of rational points modulo $q$ (see [Kah09, Theorem 9.1]). Similarly, modulo the standard conjectures, the affirmative answer to Problem 7.5 for a Fourier-Mukai pair $(X, Y)$ over $\mathbb{C}$ implies the equality of Hodge numbers of $X$ and $Y$ (see [And04, Section 7.1.2]).

To conclude the paper, we give an affirmative answer to Problem 7.3 (and hence to Problem (7.5) for the pairs discussed in Corollary 6.7.

Proposition 7.6. If $(A, B)$ is a pair of D-equivalent complex abelian surfaces, then one has $\left[\mathfrak{h}\left(A^{[n]}\right)\right]=\left[\mathfrak{h}\left(B^{[n]}\right)\right] \in K_{0}\left(\mathrm{CM}_{\mathbb{C}}\right)$ for any positive integer $n$.

The following argument is a slight modification of the proof of [Oka18, Lemma 2.1].

Proof. As we quoted above, Orlov proved that $A$ and $B$ are isogenous and hence $\mathfrak{h}(A) \simeq$ $\mathfrak{h}(B)$. To show the case $n \geq 2$, let us consider the generating series as follows.

$$
\mathbb{H}_{Z}(T):=\sum_{n=0}^{\infty}\left[Z^{[n]}\right] T^{n}=1+[Z] T+\cdots \in 1+T \cdot K_{0}(\mathcal{V} \text { ar } / \mathbb{C}) \llbracket T \rrbracket .
$$

By [GZLMH06, COROLLARY], for any smooth quasi-projective variety $Z$, there is an equality

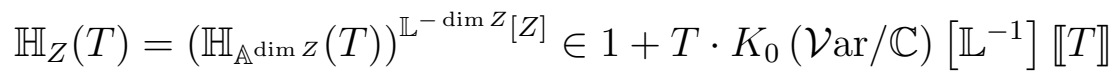

(see GZLMH06] and references therein for the notion of the power structure on $K_{0}(\mathcal{V a r} / \mathbf{k})$ ). On the other hand, since the Gillet-Soulé measure (7.8) preserves the $\lambda$-ring structures (see [Hei07, Section 4.3] and the proof of [RT15, Proposition 4.8]) and hence the power structures (see [GZLMH06, Proposition 1]), (17.10) implies the similar formula in the Grothendieck ring of Chow motives

$$
\begin{aligned}
& \mu_{\mathrm{GS}}\left(\mathbb{H}_{Z}(T)\right)=\sum_{n=0}^{\infty}\left[\mathfrak{h}\left(Z^{[n]}\right)\right] T^{n} \\
& =\mu_{\mathrm{GS}}\left(\mathbb{H}_{\mathbb{A} \operatorname{dim} Z}(T)\right)^{[\mathfrak{h}(Z)(-\operatorname{dim} Z)]} \in 1+T \cdot K_{0}\left(\mathrm{CM}_{\mathbb{C}}\right) \llbracket T \rrbracket,
\end{aligned}
$$

where $(-)$ is the Tate twist. By comparing the coefficients of $T^{n}$ in the equality

$$
\begin{aligned}
\mu_{\mathrm{GS}}\left(\mathbb{H}_{A}(T)\right) & =\mu_{\mathrm{GS}}\left(\mathbb{H}_{\left.\mathbb{A}^{\operatorname{dim} A}(T)\right)^{[\mathfrak{h}(A)(-\operatorname{dim} A)]}}\right. \\
& =\mu_{\mathrm{GS}}\left(\mathbb{H}_{\mathbb{A}} \operatorname{dim} B(T)\right)^{[\mathfrak{h}(B)(-\operatorname{dim} B)]} \\
& =\mu_{\mathrm{GS}}\left(\mathbb{H}_{B}(T)\right),
\end{aligned}
$$

we obtain the assertion.

\section{REFERENCES}

[AC12] Dima Arinkin and Andrei Căldăraru, When is the self-intersection of a subvariety a fibration?, Adv. Math. 231 (2012), no. 2, 815-842. MR 2955193

[ACGH85] E. Arbarello, M. Cornalba, P. A. Griffiths, and J. Harris, Geometry of algebraic curves. Vol. I, Grundlehren der Mathematischen Wissenschaften [Fundamental Principles of Mathematical Sciences], vol. 267, Springer-Verlag, New York, 1985. MR 770932 (86h:14019) 
[AKMW02] Dan Abramovich, Kalle Karu, Kenji Matsuki, and Jarosław Włodarczyk, Torification and factorization of birational maps, J. Amer. Math. Soc. 15 (2002), no. 3, 531-572. MR 1896232

[And04] Yves André, Une introduction aux motifs (motifs purs, motifs mixtes, périodes), Panoramas et Synthèses [Panoramas and Syntheses], vol. 17, Société Mathématique de France, Paris, 2004. MR 2115000

[Bal19] Gregorio Baldi, Some remarks on motivical and derived invariants, arXiv e-prints (2019), arXiv:1910.04733.

[Bat98] Victor V. Batyrev, Stringy Hodge numbers of varieties with Gorenstein canonical singularities, Integrable systems and algebraic geometry (Kobe/Kyoto, 1997), World Sci. Publ., River Edge, NJ, 1998, pp. 1-32. MR 1672108

[BC09] Lev Borisov and Andrei Căldăraru, The Pfaffian-Grassmannian derived equivalence, J. Algebraic Geom. 18 (2009), no. 2, 201-222. MR 2475813

[BCP17] Lev A. Borisov, Andrei Caldararu, and Alexander Perry, Intersections of two Grassmannians in $\mathbb{P}^{9}$, arXiv:1707.00534, 2017.

[BE89] Robert J. Baston and Michael G. Eastwood, The Penrose transform, Oxford Mathematical Monographs, The Clarendon Press, Oxford University Press, New York, 1989, Its interaction with representation theory, Oxford Science Publications. MR 1038279 (92j:32112)

[Bit04] Franziska Bittner, The universal Euler characteristic for varieties of characteristic zero, Compos. Math. 140 (2004), no. 4, 1011-1032. MR 2059227

[BK89] A. I. Bondal and M. M. Kapranov, Representable functors, Serre functors, and reconstructions, Izv. Akad. Nauk SSSR Ser. Mat. 53 (1989), no. 6, 1183-1205, 1337. MR MR1039961 (91b:14013)

[BL04] Christina Birkenhake and Herbert Lange, Complex abelian varieties, second ed., Grundlehren der Mathematischen Wissenschaften [Fundamental Principles of Mathematical Sciences], vol. 302, Springer-Verlag, Berlin, 2004. MR 2062673

[BLL04] Alexey I. Bondal, Michael Larsen, and Valery A. Lunts, Grothendieck ring of pretriangulated categories, Int. Math. Res. Not. (2004), no. 29, 1461-1495. MR 2051435

[BO] A. Bondal and D. Orlov, Semiorthogonal decomposition for algebraic varieties, arXiv:alggeom/9506012.

[Bor18] Lev A. Borisov, The class of the affine line is a zero divisor in the Grothendieck ring, J. Algebraic Geom. 27 (2018), no. 2, 203-209. MR 3764275

[Căl05] Andrei Căldăraru, The Mukai pairing. II. The Hochschild-Kostant-Rosenberg isomorphism, Adv. Math. 194 (2005), no. 1, 34-66. MR 2141853 (2006a:14029)

[Cau16] Thomas Cauwbergs, Splicing motivic zeta functions, Rev. Mat. Complut. 29 (2016), no. 2, 455-483. MR 3490596

[CR13] Izzet Coskun and Colleen Robles, Flexibility of Schubert classes, Differential Geom. Appl. 31 (2013), no. 6, 759-774. MR 3130568

[Dan80] V. I. Danilov, Decomposition of some birational morphisms, Izv. Akad. Nauk SSSR Ser. Mat. 44 (1980), no. 2, 465-477, 480. MR 571105

[DL99] Jan Denef and François Loeser, Germs of arcs on singular algebraic varieties and motivic integration, Invent. Math. 135 (1999), no. 1, 201-232. MR 1664700

[Dol16] Igor V. Dolgachev, Endomorphisms of complex abelian varieties, http://www.math.lsa. umich.edu/ idolga/MilanLect.pdf, April 2016.

[Efi18] Alexander I. Efimov, Some remarks on L-equivalence of algebraic varieties, Selecta Math. (N.S.) 24 (2018), no. 4, 3753-3762. MR 3848032

[Eke09] Torsten Ekedahl, The Grothendieck group of algebraic stacks, arXiv:0903.3143, 032009.

[Fal83] G. Faltings, Endlichkeitssätze für abelsche Varietäten über Zahlkörpern, Invent. Math. 73 (1983), no. 3, 349-366. MR 718935

[FW84] Gerd Faltings and Gisbert Wüstholz (eds.), Rational points, Aspects of Mathematics, E6, Friedr. Vieweg \& Sohn, Braunschweig; distributed by Heyden \& Son, Inc., Philadelphia, PA, 1984, Papers from the seminar held at the Max-Planck-Institut für Mathematik, Bonn, 1983/1984. MR 766568

[GS96] H. Gillet and C. Soulé, Descent, motives and K-theory, J. Reine Angew. Math. 478 (1996), 127-176. MR 1409056 
[GZLMH06] S. M. Gusein-Zade, I. Luengo, and A. Melle-Hernández, Power structure over the Grothendieck ring of varieties and generating series of Hilbert schemes of points, Michigan Math. J. 54 (2006), no. 2, 353-359.

[Hei07] Franziska Heinloth, A note on functional equations for zeta functions with values in Chow motives, Ann. Inst. Fourier (Grenoble) 57 (2007), no. 6, 1927-1945. MR 2377891

[Hir64] Heisuke Hironaka, Resolution of singularities of an algebraic variety over a field of characteristic zero. I, II, Ann. of Math. (2) 79 (1964), 109-203; ibid. (2) 79 (1964), 205-326. MR 0199184

[HKR62] G. Hochschild, Bertram Kostant, and Alex Rosenberg, Differential forms on regular affine algebras, Trans. Amer. Math. Soc. 102 (1962), 383-408. MR 0142598 (26 \#167)

[HL] Brendan Hassett and Kuan-Wen Lai, Cremona transformations and derived equivalences of k3 surfaces, arXiv:1612.07751 (2016).

[HLOY03] Shinobu Hosono, Bong H. Lian, Keiji Oguiso, and Shing-Tung Yau, Fourier-Mukai partners of a K3 surface of Picard number one, Vector bundles and representation theory (Columbia, MO, 2002), Contemp. Math., vol. 322, Amer. Math. Soc., Providence, RI, 2003, pp. 43-55. MR 1987738

[HLOY04] Fourier-Mukai number of a K3 surface, Algebraic structures and moduli spaces, CRM Proc. Lecture Notes, vol. 38, Amer. Math. Soc., Providence, RI, 2004, pp. 177-192. MR 2096145

[Hon18] Katrina Honigs, Derived equivalence, Albanese varieties, and the zeta functions of 3dimensional varieties, Proc. Amer. Math. Soc. 146 (2018), no. 3, 1005-1013, With an appendix by Jeffrey D. Achter, Sebastian Casalaina-Martin, Katrina Honigs, and Charles Vial. MR 3750214

[Huy06] D. Huybrechts, Fourier-Mukai transforms in algebraic geometry, Oxford Mathematical Monographs, The Clarendon Press Oxford University Press, Oxford, 2006. MR 2244106 (2007f:14013)

[IIM19] Daisuke Inoue, Atsushi Ito, and Makoto Miura, Complete intersection calabi-yau manifolds with respect to homogeneous vector bundles on grassmannians, Mathematische Zeitschrift 292 (2019), no. 1, 677-703.

[IMOU] Atsushi Ito, Makoto Miura, Shinnosuke Okawa, and Kazushi Ueda, The class of the affine line is a zero divisor in the Grothendieck ring: via K3 surfaces of degree 12, arXiv:1612.08497v1.

[IMOU19] The class of the affine line is a zero divisor in the Grothendieck ring: via $G_{2}$ Grassmannians, J. Algebraic Geom. 28 (2019), no. 2, 245-250. MR 3912058

[Ito03] Tetsushi Ito, Birational smooth minimal models have equal Hodge numbers in all dimensions, Calabi-Yau varieties and mirror symmetry (Toronto, ON, 2001), Fields Inst. Commun., vol. 38, Amer. Math. Soc., Providence, RI, 2003, pp. 183-194. MR 2019152

[Jan92] Uwe Jannsen, Motives, numerical equivalence, and semi-simplicity, Invent. Math. 107 (1992), no. 3, 447-452. MR 1150598

[Kah09] Bruno Kahn, Zeta functions and motives, Pure Appl. Math. Q. 5 (2009), no. 1, 507-570. MR 2520468

[Kaw18] Yujiro Kawamata, Birational geometry and derived categories, Surveys in differential geometry 2017. Celebrating the 50th anniversary of the Journal of Differential Geometry, Surv. Differ. Geom., vol. 22, Int. Press, Somerville, MA, 2018, pp. 291-317. MR 3838122

[KKM17] Grzegorz Kapustka, Michał Kapustka, and Riccardo Moschetti, Equivalence of K3 surfaces from Verra threefolds, arXiv e-prints (2017), arXiv:1712.06958.

[Kon09] Maxim Kontsevich, Notes on motives in finite characteristic, Algebra, arithmetic, and geometry: in honor of Yu. I. Manin. Vol. II, Progr. Math., vol. 270, Birkhäuser Boston, Inc., Boston, MA, 2009, pp. 213-247. MR 2641191

[KR17] Michał Kapustka and Marco Rampazzo, Torelli problem for Calabi-Yau threefolds with GLSM description, arXiv e-prints (2017), arXiv:1711.10231.

[KS] Alexander Kuznetsov and Evgeny Shinder, Grothendieck ring of varieties, D- and Lequivalence, and families of quadrics, arXiv:1612.07193v1.

[KS18] Alexander Kuznetsov and Evgeny Shinder, Grothendieck ring of varieties, D- and Lequivalence, and families of quadrics, Selecta Math. (N.S.) 24 (2018), no. 4, 3475-3500. MR 3848025 
[Kuz] Alexander Kuznetsov, Homological projective duality for Grassmannians of lines, arXiv:math/0610957.

[Kuz18] _ _ Derived equivalence of Ito-Miura-Okawa-Ueda Calabi-Yau 3-folds, J. Math. Soc. Japan 70 (2018), no. 3, 1007-1013. MR 3830796

[Lat18a] Robert Laterveer, On the motive of intersections of two Grassmannians in $\mathbb{P}^{9}$, Res. Math. Sci. 5 (2018), no. 3, Paper No. 29, 24. MR 3819748

[Lat18b] Robert Laterveer, On the motive of Kapustka-Rampazzo's Calabi-Yau threefolds, arXiv eprints (2018), arXiv:1808.08338.

[Lat19] On the motive of Ito-Miura-Okawa-Ueda Calabi-Yau threefolds, arXiv e-prints (2019), arXiv:1901.04812.

[Lit98] P. Littelmann, Cones, crystals, and patterns, Transform. Groups 3 (1998), no. 2, 145-179. MR 1628449

[Man19] Laurent Manivel, Double spinor Calabi-Yau varieties, Épijournal Geom. Algébrique 3 (2019), Art. 2, 14. MR 3936623

[Mar16] Nicolas Martin, The class of the affine line is a zero divisor in the Grothendieck ring: an improvement, C. R. Math. Acad. Sci. Paris 354 (2016), no. 9, 936-939. MR 3535349

[MMY19] Ciaran Meachan, Giovanni Mongardi, and Kōta Yoshioka, Derived equivalent hilbert schemes of points on $k 3$ surfaces which are not birational, Mathematische Zeitschrift (2019).

[MNP13] Jacob P. Murre, Jan Nagel, and Chris A. M. Peters, Lectures on the theory of pure motives, University Lecture Series, vol. 61, American Mathematical Society, Providence, RI, 2013. MR 3052734

[Muk81] Shigeru Mukai, Duality between $D(X)$ and $D(\hat{X})$ with its application to Picard sheaves, Nagoya Math. J. 81 (1981), 153-175. MR 607081 (82f:14036)

[Muk88] _ Curves, K3 surfaces and Fano 3-folds of genus $\leq 10$, Algebraic geometry and commutative algebra, Vol. I, Kinokuniya, Tokyo, 1988, pp. 357-377. MR 977768

[Muk92] , Polarized K3 surfaces of genus 18 and 20, Complex projective geometry (Trieste, 1989/Bergen, 1989), London Math. Soc. Lecture Note Ser., vol. 179, Cambridge Univ. Press, Cambridge, 1992, pp. 264-276. MR 1201388 (94a:14039)

[Muk99] _ Duality of polarized K3 surfaces, New trends in algebraic geometry (Warwick, 1996), London Math. Soc. Lecture Note Ser., vol. 264, Cambridge Univ. Press, Cambridge, 1999, pp. 311-326. MR 1714828

[Ogu02] Keiji Oguiso, K3 surfaces via almost-primes, Math. Res. Lett. 9 (2002), no. 1, 47-63. MR 1892313

[Oka18] Shinnosuke Okawa, An example of birationally inequivalent projective symplectic varieties which are D-equivalent and L-equivalent, arXiv e-prints (2018), arXiv:1801.09385.

[OR] John Christian Ottem and Jørgen Vold Rennemo, A counterexample to the birational Torelli problem for Calabi-Yau 3-folds, arXiv:1706.09952.

[Orl02] D. O. Orlov, Derived categories of coherent sheaves on abelian varieties and equivalences between them, Izv. Ross. Akad. Nauk Ser. Mat. 66 (2002), no. 3, 131-158. MR 1921811

[Orl05] _ Derived categories of coherent sheaves, and motives, Uspekhi Mat. Nauk 60 (2005), no. 6(366), 231-232. MR 2225203

[Plo07] David Ploog, Equivariant autoequivalences for finite group actions, Adv. Math. 216 (2007), no. 1, 62-74. MR 2353249 (2009c:14095)

[Poo] Bjorn Poonen, Mathoverflow, http://mathoverflow.net/questions/16992/ non-principally-polarized-complex-abelian-varieties.

[PS11] Mihnea Popa and Christian Schnell, Derived invariance of the number of holomorphic 1-forms and vector fields, Ann. Sci. Éc. Norm. Supér. (4) 44 (2011), no. 3, 527-536. MR 2839458

[Rød00] Einar Andreas Rødland, The Pfaffian Calabi-Yau, its mirror, and their link to the Grassmannian G(2,7), Compositio Math. 122 (2000), no. 2, 135-149. MR 1775415 (2001h:14051)

[RT15] Niranjan Ramachandran and Gonçalo Tabuada, Exponentiable motivic measures, J. Ramanujan Math. Soc. 30 (2015), no. 4, 349-360. MR 3437198

[Swa96] Richard G. Swan, Hochschild cohomology of quasiprojective schemes, J. Pure Appl. Algebra 110 (1996), no. 1, 57-80. MR 1390671 (97j:19003)

[SZ19] Evgeny Shinder and Ziyu Zhang, L-equivalence for degree five elliptic curves, elliptic fibrations and K3 surfaces, arXiv e-prints (2019), arXiv:1907.01335. 
[Tab05] Gonçalo Tabuada, Invariants additifs de DG-catégories, Int. Math. Res. Not. (2005), no. 53, 3309-3339. MR 2196100

[Tab13] _ Chow motives versus noncommutative motives, J. Noncommut. Geom. 7 (2013), no. 3, 767-786. MR 3108695

[Tre84] S. L. Tregub, Three constructions of rationality of a cubic fourfold, Vestnik Moskov. Univ. Ser. I Mat. Mekh. (1984), no. 3, 8-14. MR 749015

[Ued19] Kazushi Ueda, $G_{2}$-Grassmannians and derived equivalences, Manuscripta Math. 159 (2019), no. 3-4, 549-559. MR 3959275

[Yek02] Amnon Yekutieli, The continuous Hochschild cochain complex of a scheme, Canad. J. Math. 54 (2002), no. 6, 1319-1337. MR 1940241 (2004d:16016b)

Graduate School of Mathematics, Nagoya University, Furocho, Chikusaku, Nagoya, 464-8602, JAPAN

E-mail address: atsushi.ito@math.nagoya-u.ac.jp

Korea Institute for Advanced Study, 85 Hoegiro, Dongdaemun-gu, Seoul, 130-722, REPUBLIC OF KOREA.

E-mail address: miura@kias.re.kr

Department of Mathematics, Graduate School of Science, Osaka University, Machikaneyama 1-1, Toyonaka, Osaka, 560-0043, Japan.

E-mail address: okawa@math.sci.osaka-u.ac.jp

Graduate School of Mathematical Sciences, The University of Tokyo, 3-8-1 Komaba, Meguro-KU, TOKYO, 153-8914, JAPAN.

E-mail address: kazushi@ms.u-tokyo.ac.jp 